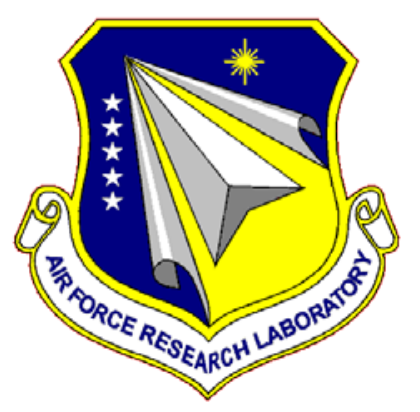

AFRL-RH-WP-TR-2014-0112

\title{
A Case Study of Human-in-the-loop for Telescope Operation
}

\author{
Vincent A. Schmidt, PhD \\ John D. Ianni \\ Judson S. Shattuck \\ Jacob A. Kern
}

Air Force Research Laboratory

August 2014

Interim Report

Distribution A: Approved for public release; distribution is unlimited.

\section{STINFO COPY}

AIR FORCE RESEARCH LABORATORY

$711^{\mathrm{TH}}$ HUMAN PERFORMANCE WING, HUMAN EFFECTIVENESS DIRECTORATE, WRIGHT-PATTERSON AIR FORCE BASE, OH 45433

AIR FORCE MATERIEL COMMAND UNITED STATES AIR FORCE 


\section{Notice and Signature Page}

Using Government drawings, specifications, or other data included in this document for any purpose other than Government procurement does not in any way obligate the U.S. Government. The fact that the Government formulated or supplied the drawings, specifications, or other data does not license the holder or any other person or corporation; or convey any rights or permission to manufacture, use, or sell any patented invention that may relate to them.

Qualified requestors may obtain copies of this report from the Defense Technical Information Center (DTIC) (http://www.dtic.mil).

AFRL-RH-WP-TR-2014-0112 HAS BEEN REVIEWED AND IS APPROVED FOR PUBLICATION IN ACCORDANCE WITH ASSIGNED DISTRIBUTION STATEMENT.

//signed//

JOHN D. IANNI

Program Manager

Battlespace Visualization Branch //signed//

JEFFREY L. CRAIG

Chief, Battlespace Visualization Branch

Warfighter Interface Division

//signed//

WILLIAM E. RUSSELL

Chief, Warfighter Interface Division

Human Effectiveness Directorate

This report is published in the interest of scientific and technical information exchange and its publication does not constitute the Government's approval or disapproval of its ideas or findings. 
The public reporting burden for this collection of information is estimated to average 1 hour per response, including the time for reviewing instructions, searching existing data sources, searching existing data sources, gathering and maintaining the data needed, and completing and reviewing the collection of information. Send comments regarding this burden estimate or any other aspect of this collection of information, including suggestions for reducing this burden, to Department of Defense, Washington Headquarters Services, Directorate for Information Operations and Reports (0704-0188), 1215 Jefferson Davis Highway, Suite 1204, Arlington, VA 22202-4302. Respondents should be aware that notwithstanding any other provision of law, no person shall be subject to any penalty for failing to comply with a collection of information if it does not display a currently valid OMB control number.

\section{REPORT DATE (DD-MM-YY) 22-08-14 \\ 2. REPORT TYPE Interim Report}

4. TITLE AND SUBTITLE

A Case Study of Human-in-the-loop for Telescope Operation

3. DATES COVERED (From - To)

1 September 2013 - 22 August 2014

5a. CONTRACT NUMBER

N/A

5b. GRANT NUMBER

N/A

5c. PROGRAM ELEMENT NUMBER 0602202F

6. AUTHOR(S)

Vincent A. Schmidt, PhD

John D. Ianni

Judson S. Shattuck

Jacob A. Kern

5d. PROJECT NUMBER

5329

5e. TASK NUMBER

11

5f. WORK UNIT NUMBER

53291103

7. PERFORMING ORGANIZATION NAME(S) AND ADDRESS(ES)

Air Force Research Laboratory (AFRL)

711 Human Performance Wing, Battlespace Visualization Branch (711 HPW/RHCV) 2255 H Street

Wright-Patterson Air Force Base, Ohio 45433-7022

9. SPONSORING/MONITORING AGENCY NAME(S) AND ADDRESS(ES)

711 HPW/RHCV (Attn: Vincent Schmidt)

2255 H Street

Wright-Patterson Air Force Base, Ohio 45433-7022

8. PERFORMING ORGANIZATION REPORT NUMBER

N/A

10. SPONSORING/MONITORING AGENCY ACRONYM

$711 \mathrm{HPW} / \mathrm{RHCV}$

11. SPONSORING/MONITORING REPORT NUMBER

AFRL-RH-WP-TR-2014-0112

\section{DISTRIBUTIONIAVAILABILITY STATEMENT}

Distribution A: Approved for public release; distribution unlimited.

13. SUPPLEMENTARY NOTES

88ABW Cleared 11/06/2014; 88ABW-2014-5151.

\section{ABSTRACT}

This paper documents a human factors assessment performed in conjunction with a small telescope research study called GOLDS -

Geosynchronous Earth Orbit (GEO) Observations with Latitudinal Diversity, Simultaneously. GOLDS included multiple distributed sites, spanning

from Northern Virginia to Maui, Hawaii, collecting electro-optical data of GEO satellites using 16-20 inch diameter telescopes. Since the authors of this report were actively involved in the telescope data collection, a significant amount of this report will focus on the first-hand experiences of the Human Effectiveness Directorate team at Wright-Patterson Air Force Base, Ohio. In addition, human factors surveys were distributed to assess human-centric issues at the other sites. The concept of operations at each site differed in some significant ways, which itself was noteworthy, but made it difficult to formulate general recommendations. For example, some sites had a high degree of autonomy while others required constant manning of the telescope. Some relied heavily on one person to operate the telescope while others used teams. These different approaches underscore the need for organizations to have facilities and procedures tailored to their capabilities and needs.

15. SUBJECT TERMS

Space situational awareness, SSA, telescope, human factors, human-in-the-loop, operations.

16. SECURITY CLASSIFICATION OF:

\begin{tabular}{|l|l|l|}
\hline a. REPORT & b. ABSTRACT & c. THIS PAGE
\end{tabular}

Unclassified

\section{Unclassified}

Unclassified
17. LIMITATION
OF ABSTRACT

SAR

\begin{tabular}{|c} 
18. NUMBER \\
OF PAGES \\
64
\end{tabular}

19a. NAME OF RESPONSIBLE PERSON

Douglas Franck

19b. TELEPHONE NUMBER (Include Area Code) 
- THIS PAGE INTENTIONALLY LEFT BLANK - 


\section{Table of Contents}

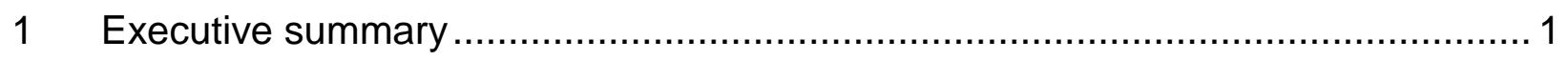

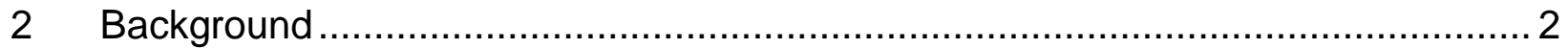

2.1 Why study the human factors of telescopes? ........................................ 2

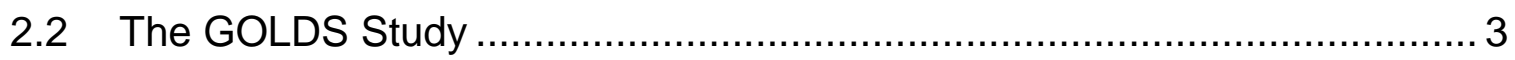

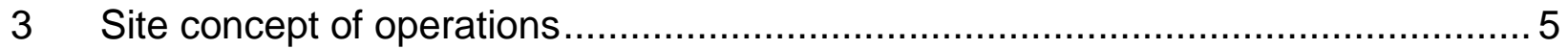

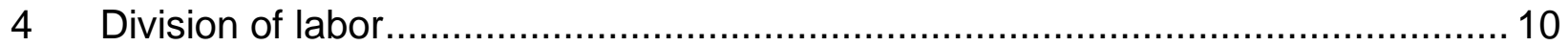

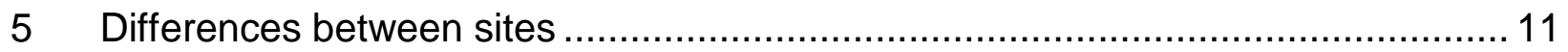

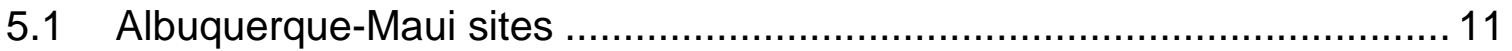

5.2 Air Force Academy sites ................................................................ 13

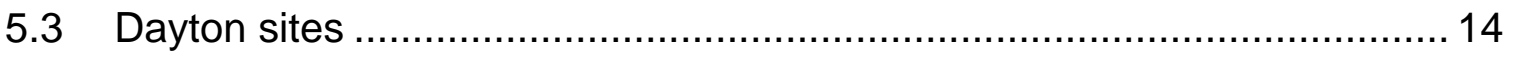

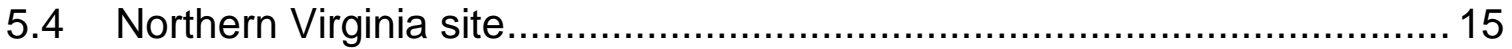

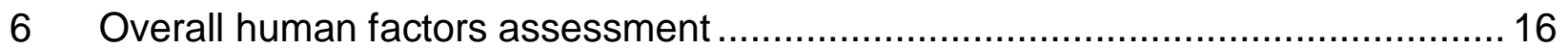

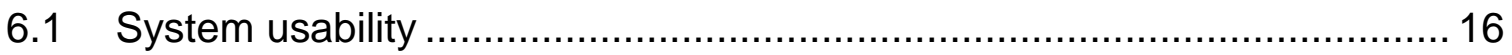

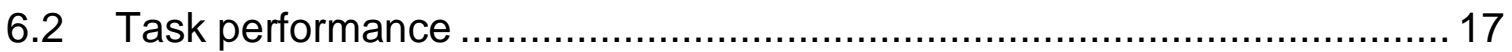

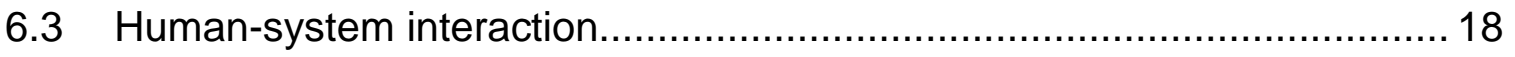

6.3.1 General theory of the observatory system ................................ 18

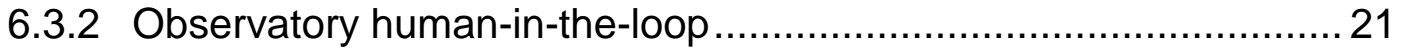

6.3.3 Multiple site human-centric issues.......................................... 23

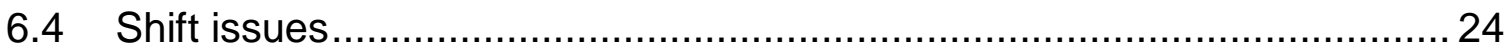

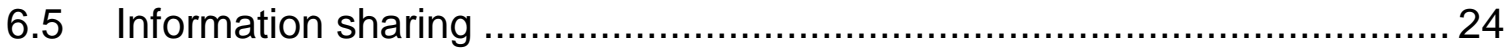

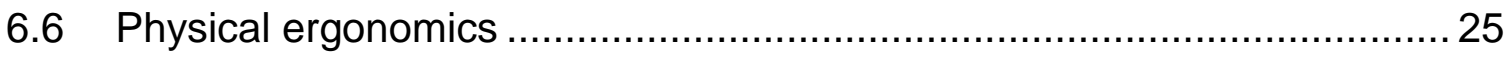

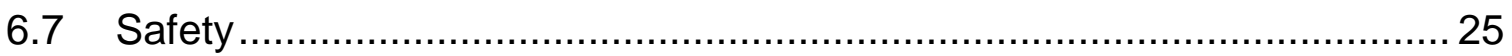

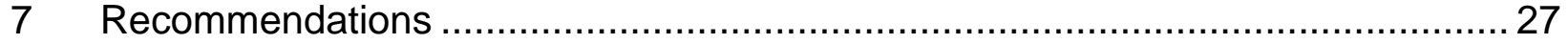

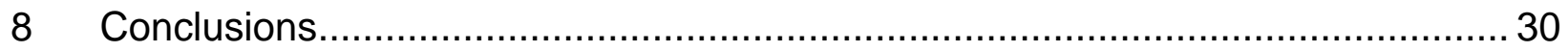

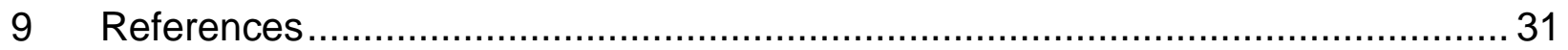

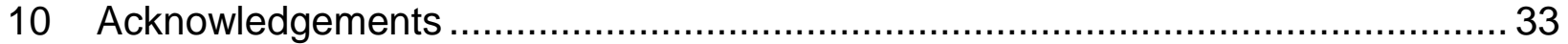

Appendix A - Site startup and shutdown procedures................................................. 34

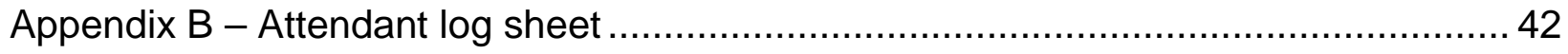




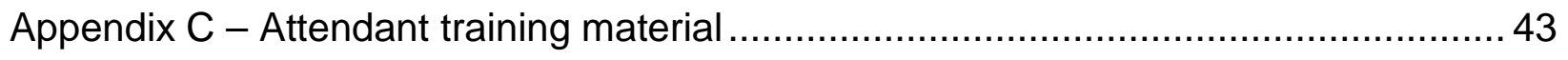

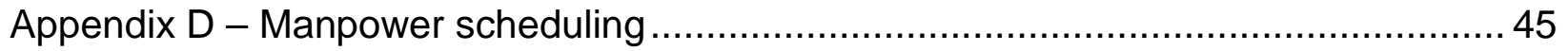

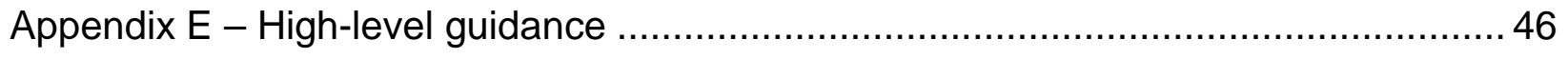

Appendix F - Situation report examples (various sites, not identified) ......................... 47

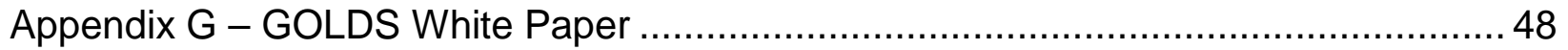

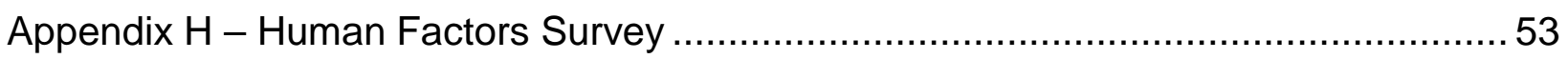




\section{List of Figures}

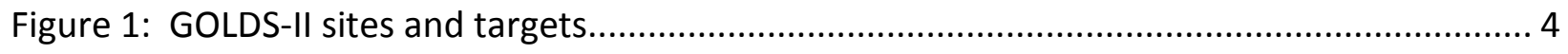

Figure 2: Telescope scissor lift in down position .......................................................... 6

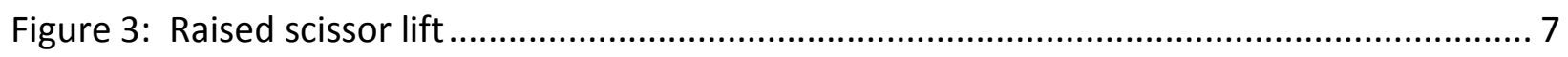

Figure 4: Human Effectiveness 17-inch telescope inside the dome....................................... 8

Figure 5: Human Effectiveness telescope's motorized dome............................................... 9

Figure 6: AMOS site on Mount Haleakala (Creative Commons photo) .................................... 12

Figure 7: USAF Academy Observatory in Colorado Springs ................................................ 14

Figure 8: Human Systems Integration areas of interest ................................................ 16

Figure 9: Usage of time during GOLDS data collections .................................................. 17

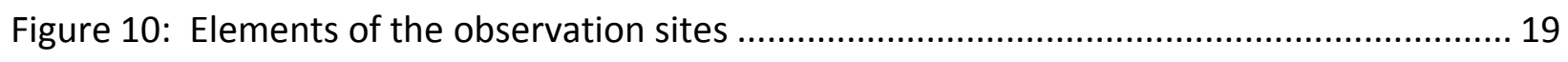

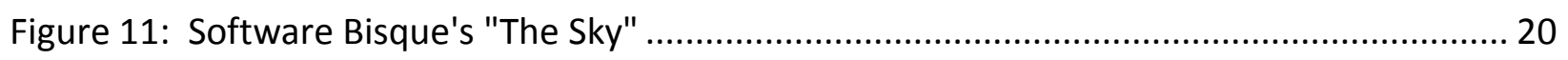

Figure 12: Software and physical interactions of the operators and attendants at RH site ....... 22

Figure 13: Processes and products for GOLDS-II Collection and Analysis. ............................ 23 


\section{Executive summary}

This paper documents a human factors assessment performed in conjunction with a small telescope research study called GOLDS - Geosynchronous Earth Orbit (GEO) Observations with Latitudinal Diversity, Simultaneously. GOLDS included multiple distributed sites, spanning from Northern Virginia to Maui, Hawaii, collecting electro-optical data of GEO satellites using 16-20 inch diameter telescopes. Since the authors of this report were actively involved in the telescope data collection, a significant amount of this report will focus on the first-hand experiences of the Human Effectiveness Directorate team at Wright-Patterson Air Force Base, Ohio. In addition, human factors surveys were distributed to assess human-centric issues at the other sites. The concept of operations at each site differed in some significant ways, which itself was noteworthy, but made it difficult to formulate general recommendations. For example, some sites had a high degree of autonomy while others required constant manning of the telescope. Some relied heavily on one person to operate the telescope while others used teams. These different approaches underscore the need for organizations to have facilities and procedures tailored to their capabilities and needs. 


\section{Background}

\subsection{Why study the human factors of telescopes?}

A small telescope can mean different things to different people. For astronomers and space professionals, "small" generally means that the primary mirror is less than one meter ( -36 inches) in diameter. Despite the implications of being small, these telescopes are often permanently or semipermanently mounted at an observing site, and the telescope system tends to include an array of assorted control and support equipment. The relatively inexpensive control hardware and software that many amateurs use for their backyard 6-16 inch telescopes are frequently the same components employed to operate telescopes up to a meter in aperture. However, since the half-meter to full-meter class telescopes are generally more capable devices, the subsystems, collection devices (cameras), and control systems tend to be more feature-rich, which makes them more complicated for operators to use.

Although the optical, electrical, and mechanical portions of the system are well-defined, the humansystem interaction issues of these machines have not received much attention. Having recently installed and used a telescope ourselves, it was evident that using the system was far from turnkey, and procedural improvements would be possible. It also became apparent that most sites refine or adjust their procedures as they determine better procedures or encounter new situations.

Therefore, the Human Effectiveness Directorate ${ }^{1}$, herein referred to as "RH", took part in the GOLDS-II research study ${ }^{2}$ to, among other objectives, review the human factors of telescope operation. This report's authors have been involved in space situational awareness (SSA) research for several years, but the focus had primarily been on the data consumer end - mainly for the Joint Space Operations Center (JSpOC). For JSpOC-related research, the focus was generally answering questions like "what is the best way to present SSA data to analysts and decision makers?" But in order to truly understand the more comprehensive SSA process, we felt it was important to understand the whole process from the sensor to the decision maker. Our hands-on experience with the telescope gave us a much more solid perspective of the overall SSA enterprise and processes.

The human factors analysis documented in this paper is based on a combination of our first-hand and observed experiences operating the RH telescope on the GOLDS study, in conjunction with the data collected via surveys from other sites involved in the study. Human factors analyses can be much more enlightening when the analyst is collocated with the work performed and, often even better, when the analyst is able to experience the work "hands on." This certainly proved to be true for this study because the RH team's findings were closely tied to their first-hand experience. In addition to human-system interfaces, we considered other areas of human performance such as training, safety, and fatigue.

The data collected from other sites was at least equally valuable. Unlike the RH site, these sites had experience on other data collection efforts so there was a considerable amount of collective knowledge to be gained. To tap this knowledge, the $\mathrm{RH}$ team e-mailed surveys (site-wide and individual surveys) to all

\footnotetext{
${ }^{1}$ The Human Effectiveness Directorate is part of the Air Force Material Command (AFMC), Air Force Research Laboratory (AFRL), $711^{\text {th }}$ Human Performance Wing (711 HPW). Specifically this research was performed by the Battlespace Visualization Branch (RHCV) which is one of four branches of the Warfighter Interface Division (RHC).

${ }^{2}$ More on GOLDS to follow; see Appendix G - GOLDS White Paper for more technical specifics.
} 
GOLDS sites including Maui, Hawaii; Albuquerque, New Mexico; Colorado Springs, Colorado; Dayton, Ohio; and the Washington, DC area of northern Virginia.

An objective of this report was to make recommendations allowing sites glean lessons learned from other observatories and, in particular, their human operators who are principal components of their respective telescope systems. Since each observatory setup was unique, it was not always possible to come up with recommendations that fit every situation but, in our opinion, the lack of standardization and the need to perform considerable on-site hardware and software integration was an important finding unto itself. Despite differences in location, climate, and specific system hardware and control software, site managers and operators should find helpful insights outlined in this report which, when put into practice, will improve the quality of their products by increasing the effectiveness of their most important component: the human.

The remainder of this section briefly describes the GOLDS study. The following two sections cover the details and lessons-learned from RH's involvement in the study. Section 5 includes information obtained from surveys returned from other sites. Section 6 introduces a collection of human factors assessments performed by the authors. Sections 7--8 provides some parting conclusions, and the appendices have supporting material such as documents used during the data collections.

\subsection{The GOLDS Study}

The GOLDS study had two separate phases ${ }^{3}$, both lead by AFRL's Space Vehicles Directorate. The common theme was to investigate the brightness and colors that satellites in GEO exhibit during the biannual glint seasons near Equinox (see Appendix G - GOLDS White Paper). GOLDS II, which was the focus of this human factors research study, had three objectives: (1) improve the calibration from the original GOLDS effort, (2) evaluate differences between tasking methodologies, and (3) collect high quality photometric data on GEO targets. Collections occurred every night, weather permitting, during the month of October 2013, including weekends. The sites experienced varying levels of success due to the differences in weather, manpower, and equipment.

GOLDS employed at least eight telescopes at five locations spanning approximately 4800 miles (Figure 1). Each night's data collection also focused on two separate target sets, East and West. This provided a respectable likelihood of observations on each night given differences in cloud cover and other conditions that could hinder operations on a particular night. For example, remnants of a hurricane took away a significant number of nights in the Washington, DC area while the Dayton sites were clear.

The telescopes had primary mirrors ranging from 16 to 20 inches in diameter. While this is not particularly small by many people's standards, it is small in comparison to the multiple meter telescopes across the world. For example, the Maui site on the dormant volcano Haleakala also enlists a 3.67-meter telescope known as the Advanced Electro-Optical System (AEOS) but that was not used for this study. [Air Force Research Laboratory Directed Energy Directorate, 2012] Even that is small compared to some newer telescopes that span over 10 meters.

${ }^{3}$ Additional GOLDS studies were anticipated but, as of August 2014, no formal GOLDS-III effort was underway. 


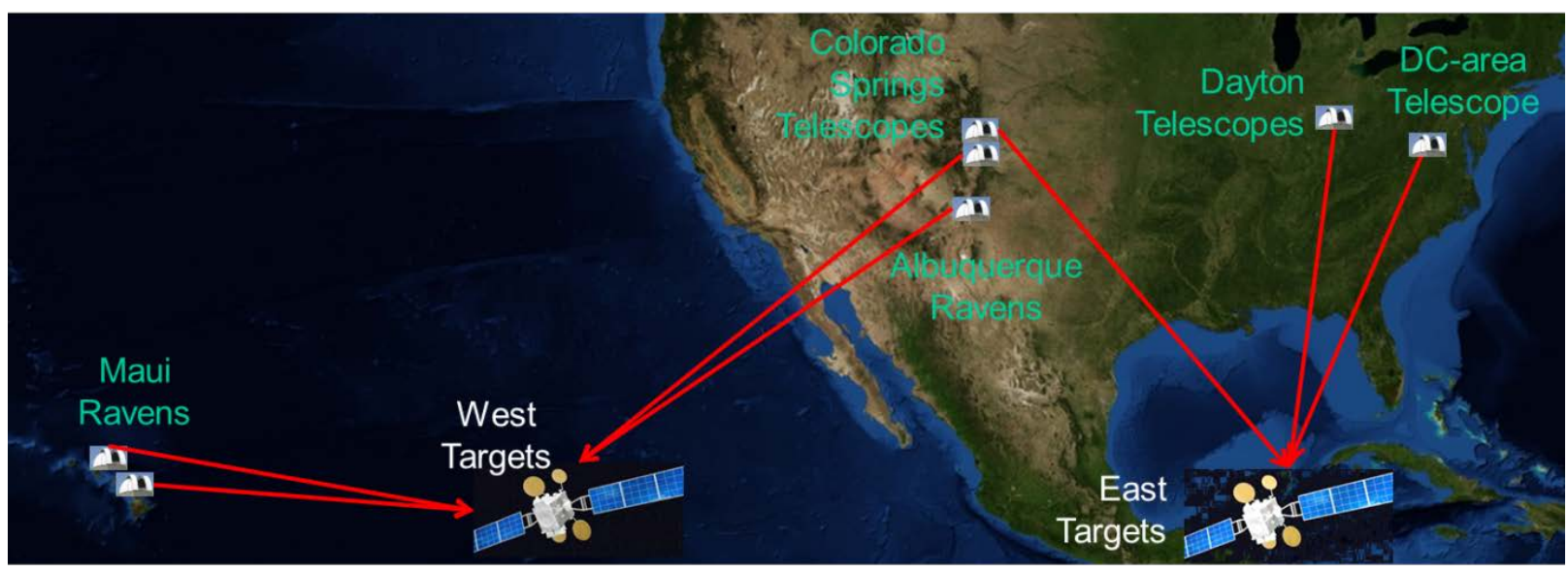

Figure 1: GOLDS-II sites and targets

The concept of operations at each GOLDS site varied significantly. Some were one person operations while others (e.g., the RH site) had a few operators who could fully operate the telescope, and a pool of attendants who manned the telescope overnight. Some sites collected data on a regular basis outside of the GOLDS study (e.g., the Maui site) while others were in use much less often.

The levels of autonomy at each site differed as well. The Albuquerque site, for example, had a high degree of autonomy giving them the ability to collect data without overnight manning. All sites used autonomy to some extent especially for camera operation which included capturing images at regular intervals and changing filters (alternating between red and blue). The automation aspects were of interest to $\mathrm{RH}$ because the directorate researches how humans interact with autonomous systems such as unmanned aerial vehicles. Other $\mathrm{RH}$ automation research includes trust of automation, optimal levels of automation for certain applications, and how to maintain the human's readiness should they need to engage. 


\section{Site concept of operations}

This section describes the configuration and concept of operations for RH's 17" PlaneWave telescope used in the GOLDS study. This was the first major data collection effort performed with the relatively new telescope at Wright-Patterson Air Force Base (Figure 2 and Figure 3) [Shattuck, et al 2012]. Although some of the operational and setup guides had been written before GOLDS, many changes were made as the study progressed and issues were identified. The documents used on this effort - such as the telescope startup and shutdown procedures and training documentation - are provided as appendices in this report.

The $\mathrm{RH}$ site included the following hardware and software:

\section{$\underline{\text { Hardware }}$}

- Telescope - PlaneWave 17" CDK equatorially mounted on a Mathis Instruments MI-500 with MI750 fork arms.

- Camera - SBIG 8300M monochrome camera with 8.3 megapixels (3326 x 2504 pixels)

- $\quad$ Filters - 8-filter wheel; only red and blue filters were used for GOLDS-II study

- Dome - 2.5-meter ObservaDome with custom rotation control system (AKD drive hardware)

\section{$\underline{\text { Software }}$}

- Graphical User Interface - The Sky X Pro

- $\quad$ Telescope drivers - STI (SiTech mount interface and ASCOM drivers)

- Focuser - PWI (PlaneWave Hedrick focuser interface and ASCOM drivers)

- Camera control - MaxImDL (preferred commercial camera control software; required for autofocus and advanced mount model configuration)

- Dome control - custom Python program written by RH personnel

Figure 2 shows RH's telescope resting on the lift platform in the lowered state. The portable pier is bolted to the railing of the lift so it will not move when the lift is raised into the dome or lowered to the floor. The platform is large enough to comfortably accommodate 2-3 people, and there is a remote keyboard, mouse, and monitor (barely visible at the center of the figure) which duplicates the interfaces used to control all aspects of the system in the main room. 


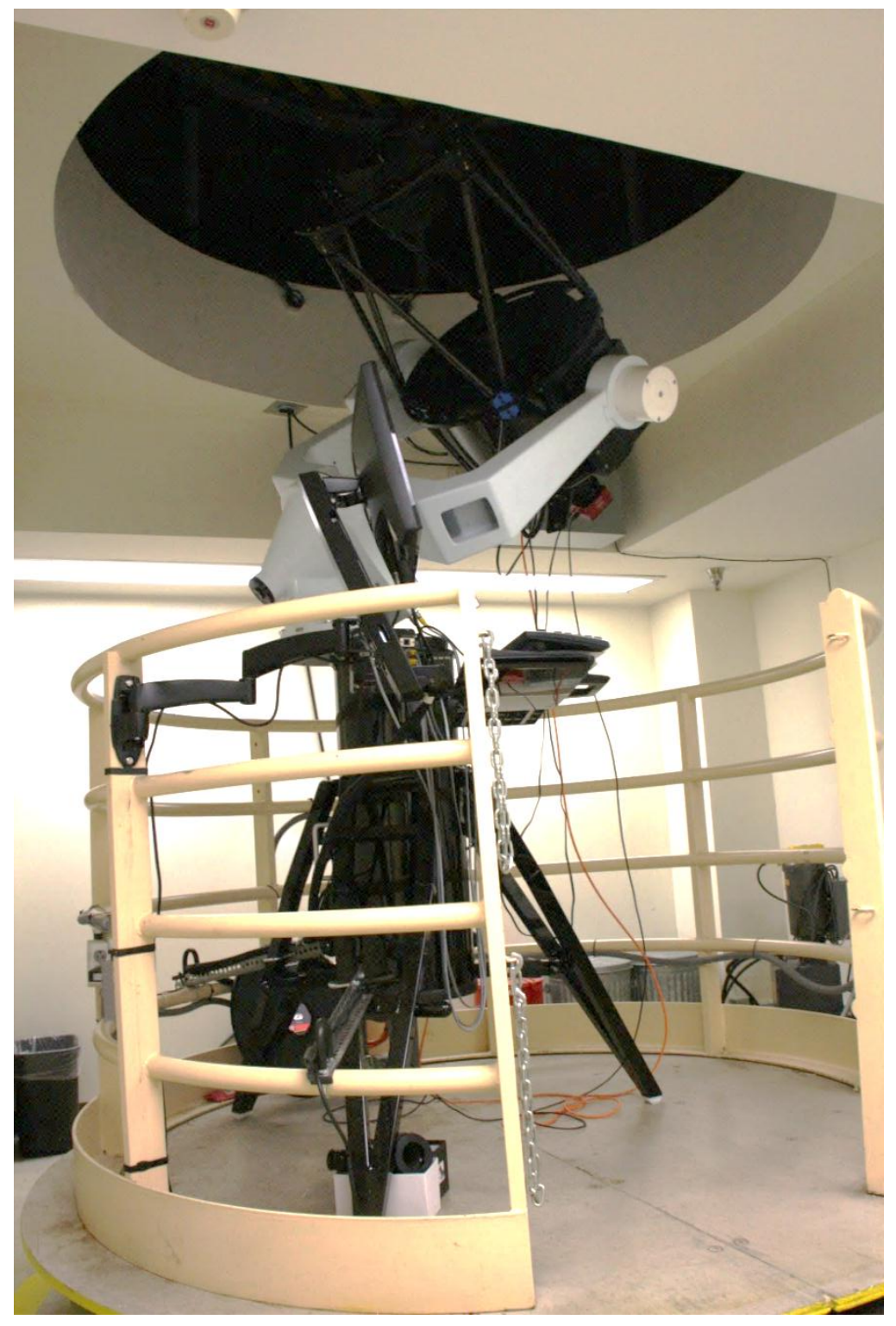

Figure 2: Telescope scissor lift in down position

The telescope and control computer were raised on a hydraulic scissor lift platform for collections (Figure 3). This raising telescope appears to be quite rare in the general astronomy community. We were originally concerned that the lift platform could introduce motion impacting the telescope data. This did not turn out to be the case since our collections seems to be quite clear.

Distribution A: Approved for public release: distribution is unlimited. 88AB/PA Cleared 11/06/2014: 88ABW-2014-5151. 


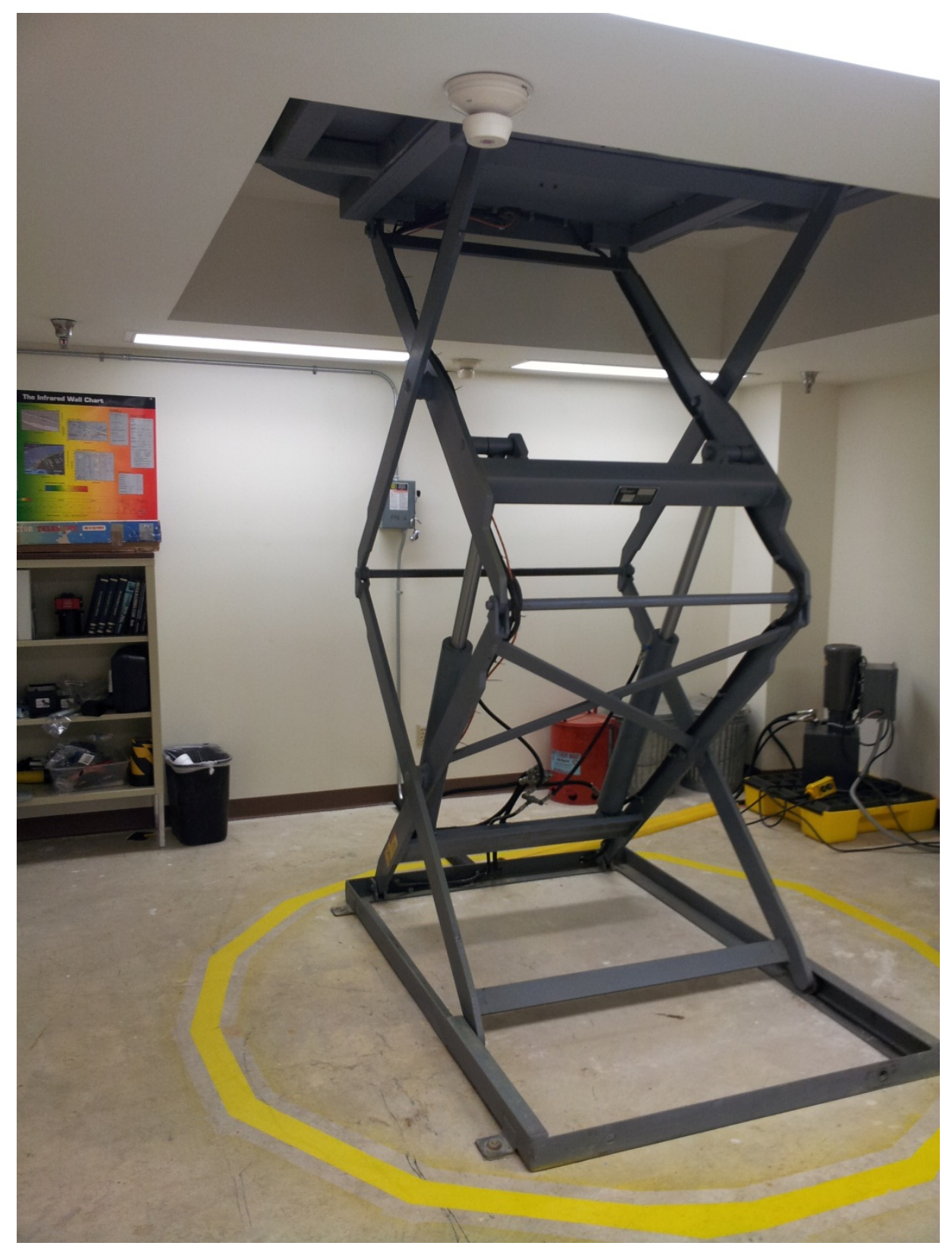

Figure 3: Raised scissor lift

The figure shows the scissor lift fully extended, necessary to allow the telescope to peer through the dome shutters. The control computer (not shown, just out of the field of view to the right) controls all telescope operations. A remote monitor, mouse, and keyboard are also provided on the platform with the telescope; an operator riding the lift could control all aspects of the system from either location.

When the telescope was raised for observing it was normally out of view of the operator (Figure 4) except when they stayed on the platform as it raised. The operator would ride the platform up to open the dome door and make some adjustments to the telescope and perform dome alignment.

Distribution A: Approved for public release: distribution is unlimited. 88AB/PA Cleared 11/06/2014: 88ABW-2014-5151. 
The fact that the telescope was out of view for normal operation was seldom an issue, however if adjustments were needed to the dome, the operator would need to make their way to the roof to see what was happening. This required going down stairs, through hallways, up a flight of stairs, and crossing a couple sections of the roof. On one occasion, for example, glare from the moon was an issue so the dome was moved to shade the light. In addition, sudden changes in the weather could not be monitored from the telescope's control room so it was necessary to check weather by leaving the control room and walking to a nearby balcony. One consideration was to mount a web camera somewhere in the dome to provide some situational awareness to the operators and attendants, but the webcam we used wasn't capable of getting a good picture of the environment in such a low-light setting.

Figure 4 shows a view of the telescope as seen from the outside of observatory dome. The dome (Figure 5 ) is positioned on the corner of a 3-story building, and has an unobscured (horizon-limited) view of most of the sky. A portion of the eastern sky is limited to views 35 degrees or more above the horizon, and the small sections of the southern sky are blocked by other buildings and structures.

Although the weather was excellent the month of the GOLDS study, Ohio weather conditions are often more of an issue than local lighting for telescope data collections. Since the weather can change rapidly, the operators and attendants checked the weather conditions regularly to prevent damage to the telescope.

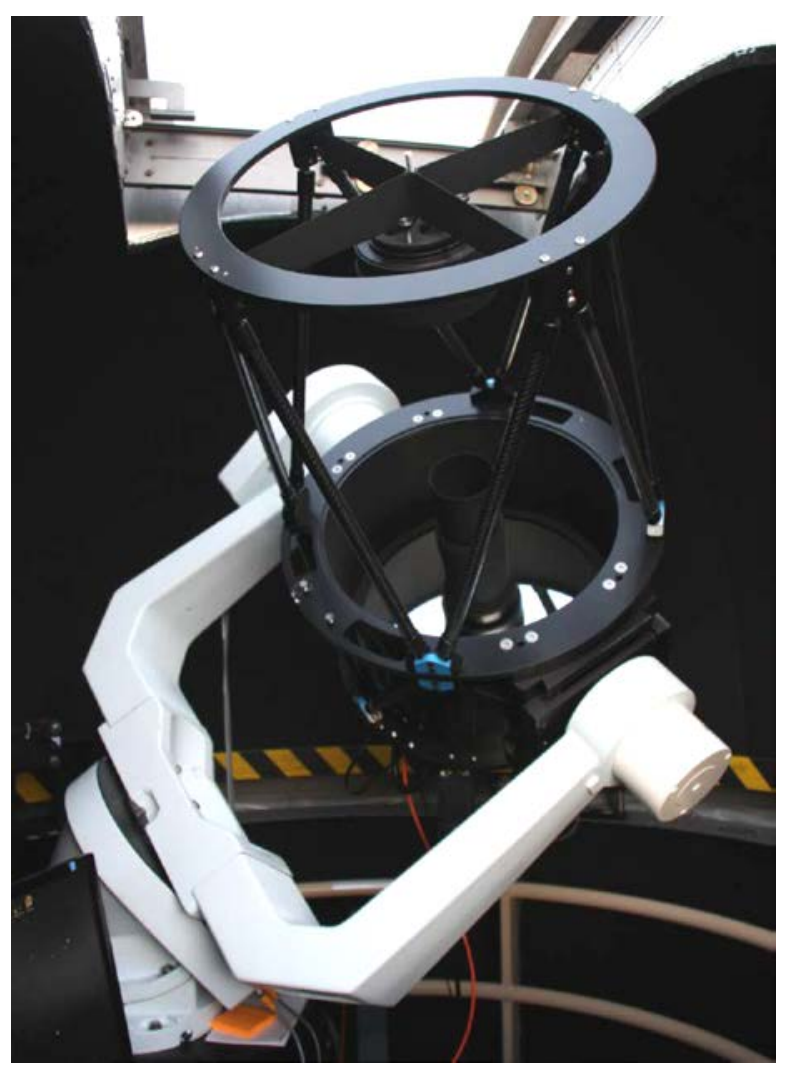

Figure 4: Human Effectiveness 17-inch telescope inside the dome

Distribution A: Approved for public release: distribution is unlimited. 88AB/PA Cleared 11/06/2014: 88ABW-2014-5151. 


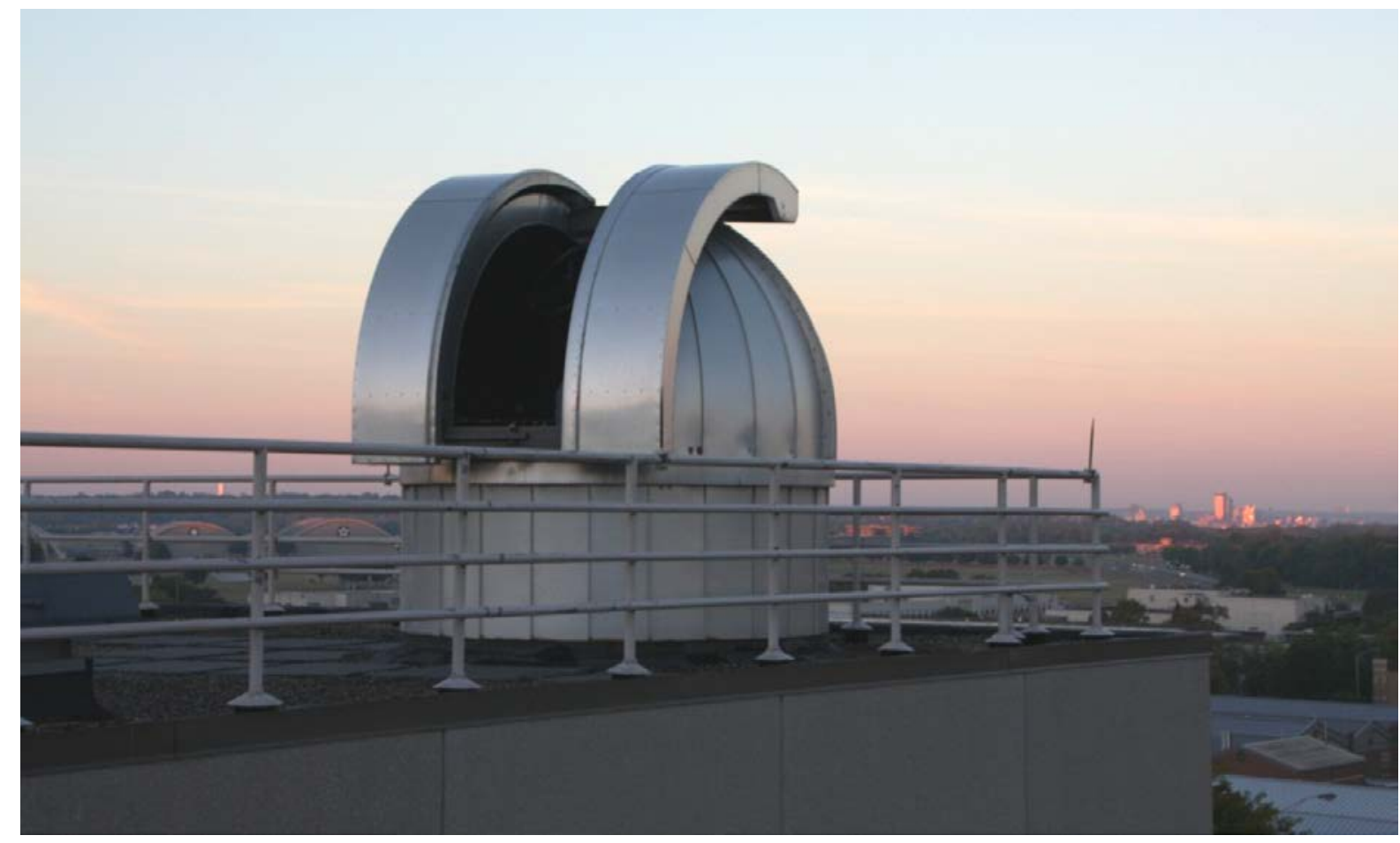

Figure 5: Human Effectiveness telescope's motorized dome

Distribution A: Approved for public release: distribution is unlimited.

88AB/PA Cleared 11/06/2014: 88ABW-2014-5151. 


\section{Division of labor}

Although not typical for GOLDS, the RH site employed nine people who could be scheduled to help with the GOLDS effort. This ensured that individual's shifts could spread out over the month as to not affect on- and off-duty obligations and sleep patterns too drastically. In addition to relieving individuals from excessive hours and shifts, this schedule also allowed those with no previous experience to attend to the telescope during steady-state operation.

The site employed a pool of four operators and five volunteer attendants (including eight civilians and one military), all of whom worked for $\mathrm{RH}$. The operators, who also were periodically scheduled for shifts as attendants, took turns starting the system in preparation for steady-state operation throughout the month.

On a typical night, the operator arrived around 2030 (8:30PM Eastern Standard Time) - approximately an hour and a half prior to the start of data collection - to get the telescope system ready for data collection in a "steady state" mode. If a separate attendant would be taking over for the overnight collection, there would be an approximate 30 minute overlap for a briefing and, if this was an attendant's first night, training. Once an attendant was settled into the data collection mode, the operator could leave for the evening. Sometimes the operator functioned as the attendant, so there was no need for overlapping shifts.

We found that attendants with no previous experience had no issues handling the overnight manning or performing the full shutdown. A simplified version of the shutdown procedure was provided to attendants in case they did not feel comfortable with the full procedure; however all of the attendants were able to perform the full shut down of the hardware, software and facilities. See "Appendix A - Site startup and shutdown procedures." The simplified shutdown would merely put the system into a "safe" state, but leave the devices fully powered; an operator would shut the system down the rest of the way the following morning.

Perhaps due to the round-robin manning, fatigue did not turn out to be a significant issue for either operators or attendants. However, on one occasion an operator, who was also acting as an attendant two nights in a row, overslept the second night. This was understandable but unfortunate because the weather was clear on the night that was missed. With the number of attendants available, it would have made more sense not to schedule anyone two nights in a row. 


\section{Differences between sites}

So far we have primarily discussed lessons learned at the $\mathrm{RH}$ site but there were probably even more lessons to be learned from the other GOLDS sites. Some of these sites have considerable experience in satellite observation so they already had developed best practices that could, in some cases, be generalized. Therefore surveys were distributed (Appendix G - Human Factors Survey) and interviews were conducted in order to assess the various human-in-the-loop issues and possibly obtain some best practices. In addition, requests were made for documentation such as the sites' operational guides, training manuals, and the like. This gave us a reasonable idea of the sites operations.

In the two surveys (site and individual), questions were developed to pull information about setup procedures, operational procedures, scheduling, manning, training, documentation, fatigue, errors, equipment issues, and others. Manning issues involved whether the site had separate operators and attendants, how many of each were in the pool, and so on. It is also worth noting that each site was supposed to submit weekly situation reports via e-mail to the rest of the GOLDS team.

\subsection{Albuquerque-Maui sites}

The three Raven telescopes at the Albuquerque and Maui sites were operated by two individuals located in Albuquerque. One operator was responsible for both of the Maui Raven systems and the other operator for the Albuquerque Raven system. Both operators were already familiar with the systems hardware and software. Since they were both involved in the first GOLDS data campaign and have multiple years of experience, they were also familiar with the operational procedures and requirements of the study. Both operators also performed maintenance on the Albuquerque system just days prior to the start of the study. While onsite, they collected data on various satellites, performed calibration and provided this to a researcher who reviewed and approved the data.

The Maui site (Figure 4), namely the Air Force Maui Optical and Supercomputing (AMOS) observatory, is situated at the top of the domain volcano Haleakala. The site contains several electro-optical telescopes including the 3.67-meter telescope, known as the Advanced Electro-Optical System (AEOS), which is the country's largest optical telescope designed for tracking satellites. Although the taskings came from Albuquerque, there were people on site for data retrieval and system maintenance. 


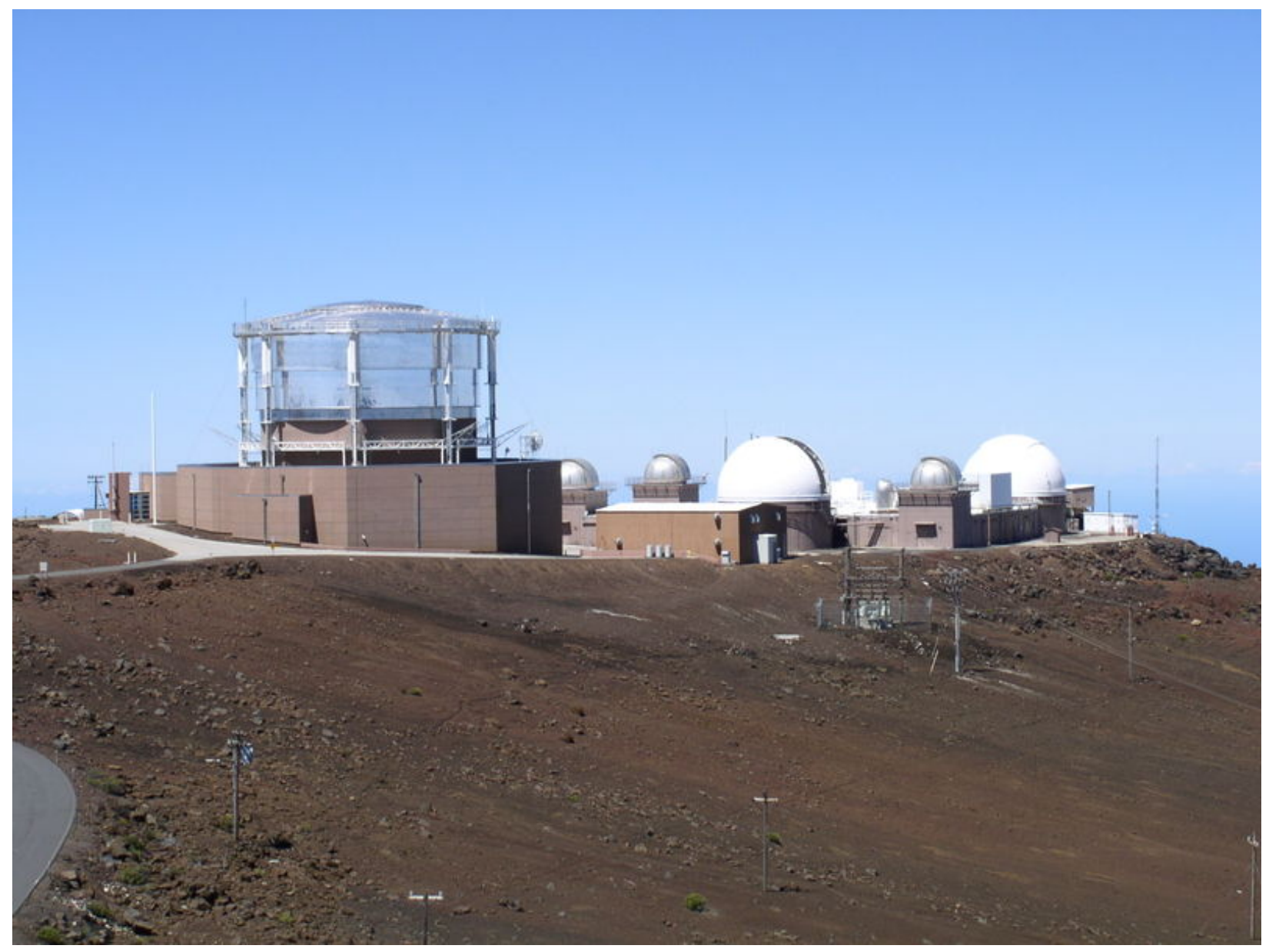

Figure 6: AMOS site on Mount Haleakala (Creative Commons photo)

These systems were highly autonomous and did not need to be attended overnight. The element sets and tasking files were uploaded by the operator to the computer and the custom software used for autonomous operations was initialized. The computer was always locked by the operator prior to leaving the site (or prior to closing out of a remote computer session in the case of the Albuquerque raven). The custom software that runs the systems autonomously runs a shutdown script at the end of the observational period. It closes the dome, parks the dome and mount, and turns off the camera cooler. Everything was left powered on since the hardware was connected to power distribution units (PDUs) which were powered with an uninterruptible power supply (UPS).

Through the surveys, the operators provided the procedures that they typically followed:

1. Retrieve 2-line orbital element sets ("elsets") from Space Track website (space-track.org).

2. Create tasking file for each telescope using the current elsets retrieved.

3. Upload the elsets and tasking files to each of the computers that would run the telescope systems.

4. Retrieve the data from each system on the next day.

5. Visually inspect data and log any anomalies or errors that may have occurred in an internal log the team keeps. 
6. Provide status report of data collection for all three systems to the GOLDS leads via email.

7. Upload data for all three systems to the drop box and let the GOLDS lead know that the data was transferred there.

Custom software was used to generate tasking files. A tasking file needed to be created for each system. First, the elsets were retrieved from the Space Track website by the operator. Then tasking was created with these elsets which took about 8 minutes to create per file. After this, the tasking file was uploaded to each computer collocated with the telescopes. For the Maui systems, the operators drove to the site, about 3 minutes away from their work location. For the Albuquerque site, a secure virtual private network connection was used to transfer the elset, tasking, and data daily.

The data collected was retrieved from the system the next day by the operators. This was done around 1:00 PM local time. For the Maui systems, it took about 15 minutes to transfer the data to the hard drives for both systems. For Albuquerque, it took about an hour to transfer the data back to Maui.

The data was visually inspected by the operators. Status of how the weather appeared from the images was then reported to the GOLDS leads via email. Comments were also included regarding satellite saturation and any camera exposure times that should be changed for the next observation period. Finally, the data was zipped and transferred to a drop box that the GOLDS lead and the Raven operations team shared. The lead then retrieved the data from the drop box and deleted the files daily to make room for the next day's data.

\subsection{Air Force Academy sites}

In contrast to the high automation at the Albuquerque site, the United States Air Force Academy (USAFA, Figure 5) telescopes were highly manual. Two Ritchey-Chrétien telescopes were employed:

1. 16-inch f/8.2 (DFM Engineering). This telescope was mounted in Colorado Springs for both GOLDS efforts. Operators and attendants were located in a separate control room from the telescope.

2. 20-inch $\mathrm{f} / 8.1$ (RCOS). This telescope, housed in a mobile trailer, was located at Plentywood, Montana on the original GOLDS effort but was moved to Colorado Springs near the 16-inch for GOLDS-II. The dome was manually opened and closed. The only automation was the controller which could slew the mount to the appropriate position. All pointing commands, camera commands, and tasking decisions were manual. The front half of trailer enclosed the 3 operators while the back half containing the telescope mount had removable walls. 


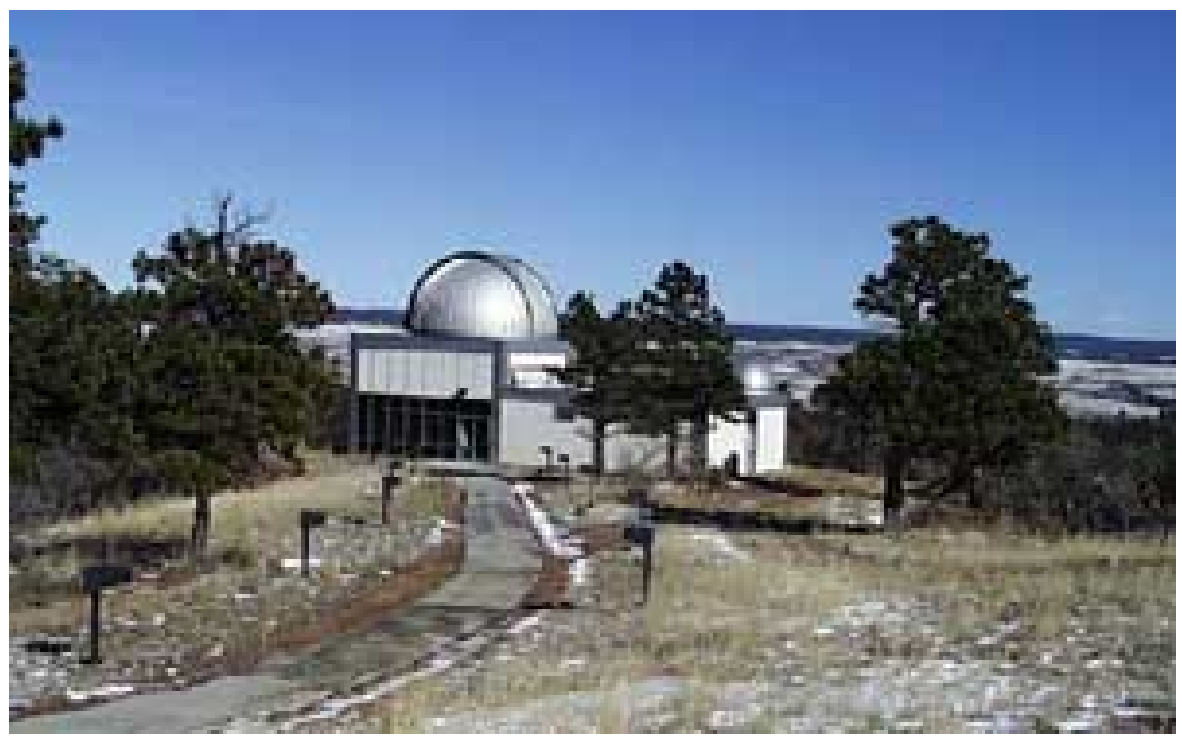

Figure 7: USAF Academy Observatory in Colorado Springs

For GOLDS II, the site employed one person per telescope each night and the manning was determined by availability. Of the eight individuals available to work the telescopes, five were operators with experience operating telescopes and three lieutenants with no previous satellite observation experience.

For the Plentywood collection, three people rotated every 30 minutes through different roles or "stations," diminishing boredom and fatigue while increasing experience and overall SA. These stations included:

- $\quad$ Operator - who sat at a computer that controlled slew, camera, and data management

- $\quad$ Tasker - who instructed operator on what to look at and for how long. (Very complex job during competing glints)

- $\quad \underline{\text { Rest }}$ - including personal time or error check other two jobs

\subsection{Dayton sites}

In Dayton, Ohio, there were three telescopes located in three different telescopes on Wright-Patterson Air Force Base. In addition to the RH telescope, there was a telescope at the Sensors Directorate (RY) building and another at the Air Force Institute of Technology (AFIT). The Dayton area was relatively clear in the month of October 2013, so a significant amount of data was collected.

The RY site used only one operator for the GOLDS campaign. He performed all of the set-up, data collection, closing-up, and system maintenance for the campaign.

AFIT collected data with their telescope during this time period but did not officially participate in the GOLDS study. They used only one operator/attendant who occasionally had duties in the mornings following his overnight telescope shifts. He was essentially self-taught using his personally owned telescope that was a different model from the AFIT telescope. Many of the procedures were adapted from collection to collection. The scope was located in a small detached building without environmental controls (heat or cooling). The operator/attendant needed to stay with the scope, thus was exposed to night's weather while performing data collections. 


\subsection{Northern Virginia site}

The Northern Virginia site in the Washington, D.C. area, also known as "East Coast", was located at The Analytic Sciences Corporation (TASC) headquarters in Chantilly, Virginia. The site had only one operator who was a Senior Optical Systems Engineer. For GOLDS-II he was only able to collect data one full night and small segments (e.g., 15 minutes) on other nights due to the remnants of hurricane Karen and some equipment failures. The site apparently had partially automated collection capabilities. No operator or site surveys were returned from this site. 


\section{Overall human factors assessment}

In this section we will examine several areas of human-system integration (HSI) observed on the GOLDS effort. A subset of the HSI areas of interest outlined in Air Force Instruction 10-601 (April 2006, see Figure 8) was used. Some of these issues may be unique to the RH site, but similar issues may be experienced at other sites. The uniqueness of each site's setup was due to a wide variety of factors, including available facilities, budgets, needs, frequency of use.

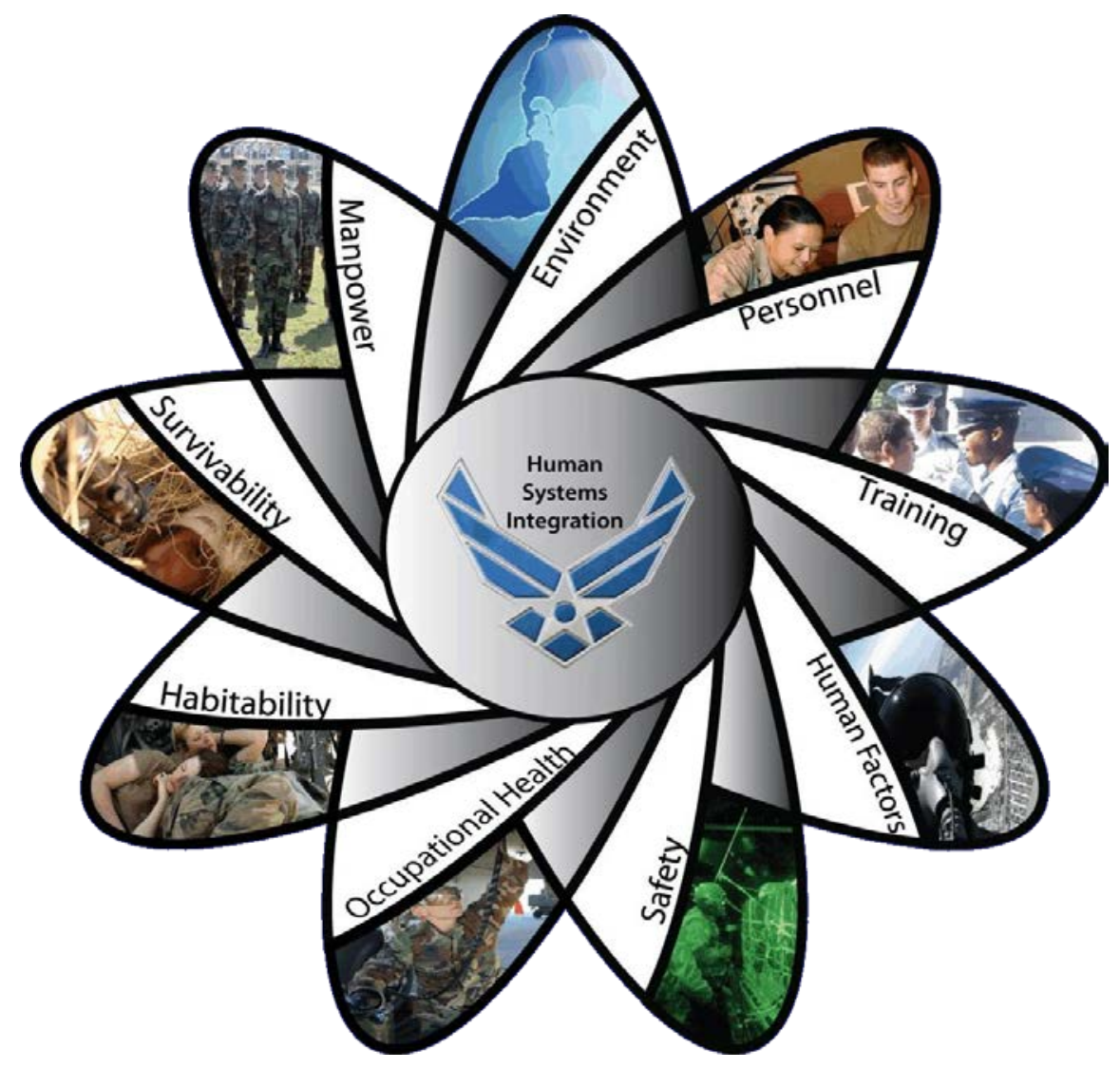

Figure 8: Human Systems Integration areas of interest

\subsection{System usability}

Usability at each site differed considerably. Some sites spent considerable effort to ensure personnel who were generally unfamiliar with these complex systems would be comfortable with basic operations by marking and labeling frequently used operations and components, as well as providing comprehensive documentation. Other sites included limited documentation, or relied on accessibility to system experts for many functions.

Usability issues for RH's telescope operation differed between the operators, who performed setup and calibration, and attendants, who performed overnight monitoring. The operators needed a fairly strong mental model of telescope theory and somewhat detailed knowledge of specific setup requirements: several software packages needed to be used for telescope control, camera control, dome control, and related systems. Although most of these packages were linked to provide data sharing and automated communication, they still needed a reasonable amount of human interaction to get them to running in concert. 
Unlike the telescope operator, the attendant did not need an advanced understanding of all system components. The attendant's job primarily involved monitoring the CCD camera's exposures of selected satellites. Specifically, this would involve using a computer mouse to draw boxes around the areas on the screen returned by the GEO satellites being observed, and inspect the computed information about the image pixels to ensure the images were not exposure-saturated. Since this was a GEO satellite data collection, there was no need to slew the telescope in most cases. Camera and filter control was highly automated by the MaxImDL camera control software.

\subsection{Task performance}

The concepts of operations and procedures also differed at each site. Each location had a different pool of people involved in running their telescope(s). These individuals had varying levels of experience, and used different telescopes, cameras, and software setups.

The RH survey included questions about how time was being used during the collection nights. The results of this survey are graphically depicted in Figure 6 . Although we know all of the operators and attendants were highly motived professionals, a great deal of their time was spent on non-collectionrelated activities, such as checking e-mail and media usage. This is consistent with activities that involve only sparse human involvement.

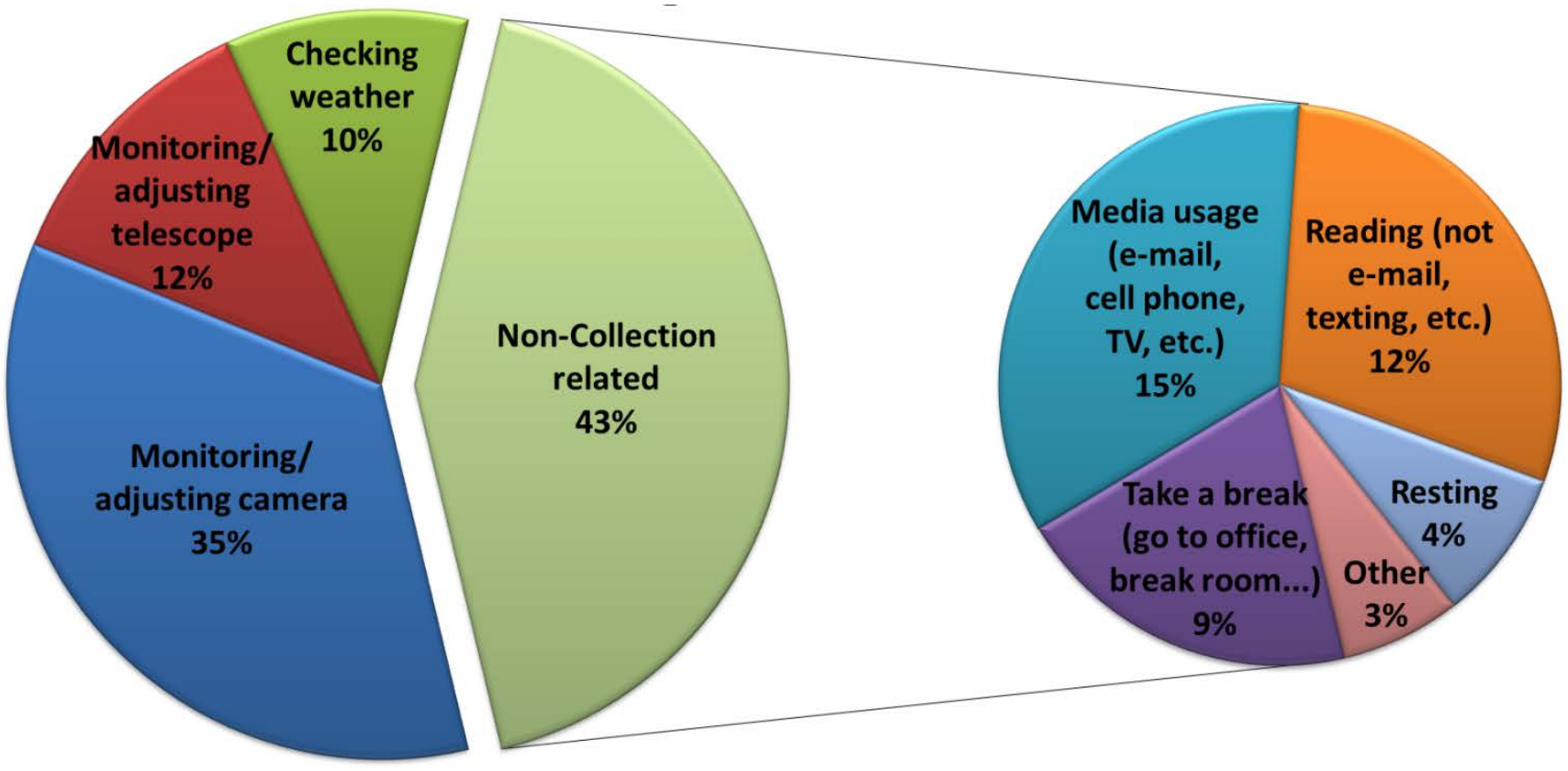

Figure 9: Usage of time during GOLDS data collections

In addition to sparse task performance, the attendants also had repetitive tasks to perform throughout the night. Repetitive tasks are often good candidates for automation since humans have a limited attention span, and repetition can cause boredom, lead to mistakes, and introduce fatigue and other issues. The most repetitive task at the $\mathrm{RH}$ Site was the monitoring of camera exposures. This task involved drawing a box around the satellite's camera image and checking the exposure. If the exposure was outside of the upper or lower thresholds determined by the GOLDS lead, camera exposure adjustments would be made.

Another repetitive task was checking the weather. At the $\mathrm{RH}$ Site, it was not possible to see weather changes during collects because the dome was hidden by the floor of the scissor-lift and the attendant's 
collection area had no windows. Therefore the attendant was encouraged (required!) to periodically leave the collection area to walk outside. Most attendants felt this was a welcome break from the monotony of monitoring camera exposure details.

It is not surprising that more of the attendant's attention was required for monitoring and adjusting the camera than the telescope. Since this was a GEO data collection, the satellites did not drift much from the camera view so little, if any, adjustments to the telescope were needed. Much of the time, the camera exposure remained fairly stable but, often around 0200-0300 local time (2:00 to 3:00 AM EDT), the exposures would spike beyond the upper exposure limits, and camera exposure corrections would be made.

\subsection{Human-system interaction}

\subsubsection{General theory of the observatory system}

In this section we will examine the RH observatory system, making note of where human interaction occurs. Consider the abstract view of an observatory system as shown in Figure 7, where the system includes a host of components characterizing the capabilities of the observatory. In this figure, we've identified the most significant components, shown so the area each component occupies in the diagram roughly represent its relative importance in the GOLDS-II study. Overlapping areas indicate cases where exceptionally close relationships exist between components. The following paragraphs describe the features of these components as they influence (or are influenced by) human interaction. 


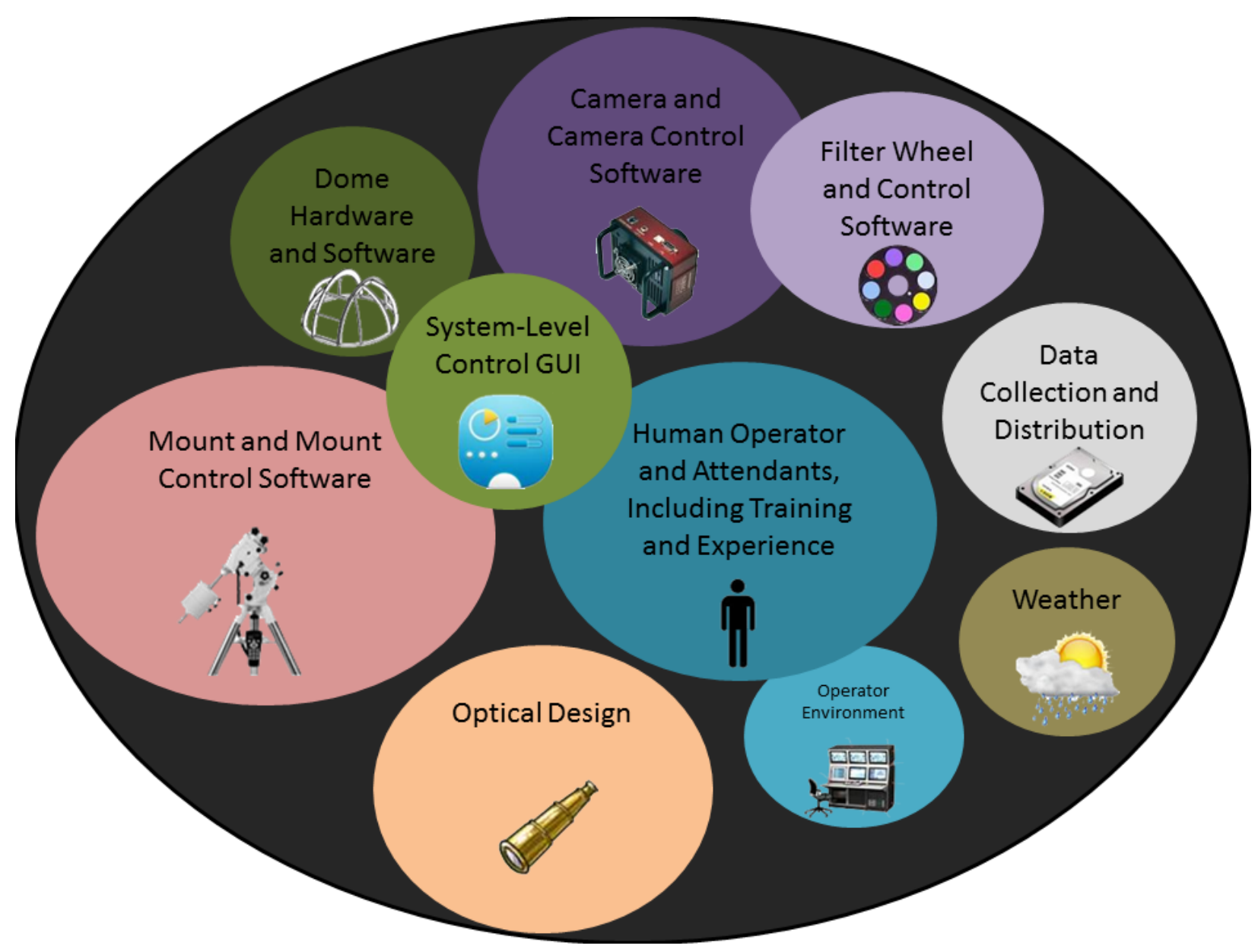

Figure 10: Elements of the observation sites

Many component subsystems comprise a complete subsystem. Even to a non-astronomer, it is obvious that the mount, mount controller, and optical designs are significant contributors to a telescope system's ability to engage in successful operations such as visual observation and digital data collection. To many, the degree to which the other components support the mission is not as obvious.

Like most contemporary complex systems, observatory systems consist of an often-complicated interaction of software parts. Aside from the observatory control systems that rely on custom software, there are several well-developed, reasonably inexpensive, and popular system-level graphical user interfaces (GUIs) controlling most telescope and observatory operations. This means that astronomers can frequently feel comfortable using a variety of telescopes because the GUI front-end is already familiar, even though the underlying hardware systems may differ. One example of such a GUI control system is Software Bisque's "The Sky" (Figure 8). 


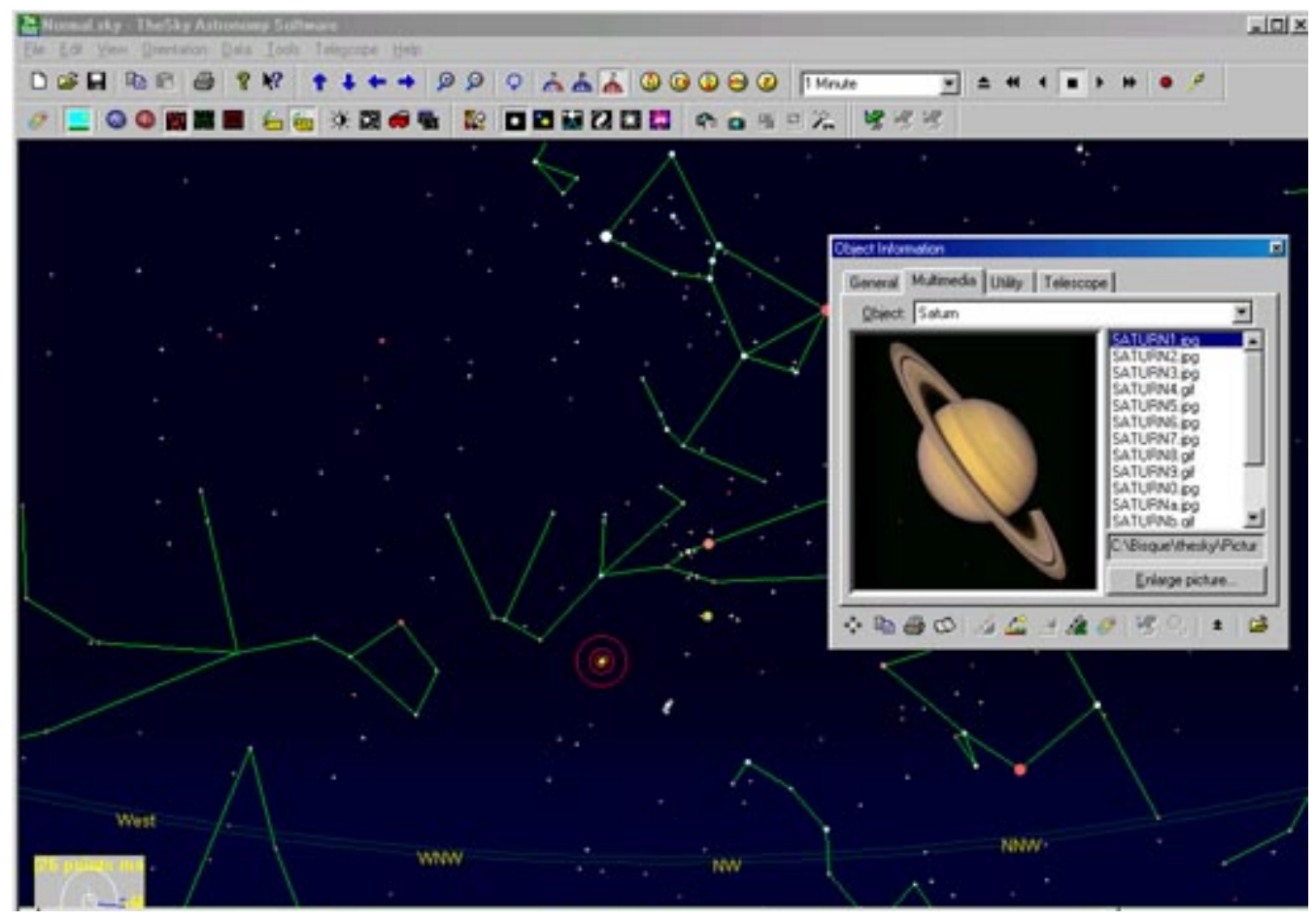

Figure 11: Software Bisque's "The Sky"

The control GUI is generally the primary mechanism through which the human operator exercises system control. Once the GUI initialization and setup has been completed (which is specific, and unique to an individual observatory configuration), the mount, dome, camera, filter wheel, and other subsystems can be controlled, and even automated, via a common and familiar interface. A degree of training and experience will be required to make the operator comfortable and efficient using this GUI, but once this familiarity has been achieved, observatories with different hardware components controlled by the same GUI can be more easily managed by the operator. Even if the controls differ between observatories, knowledge obtained while using one control system frequently transfers to using different control systems, since the terminology is similar, and the functionality of the subsystems is compartmentalized and presented in similar ways.

One important factor is the operator's environment. In some cases, such as for roll-off roof observatories and completely portable setups, the operator and the entire telescope system is exposed to the weather (including cold temperatures, windy conditions, and unfortunate interactions with light sources). In some cases, the operators might be in semi-protected environments, where a non-permanent control station is located close to the telescope gear, providing the operator with some degree of environmental protection. The Raven telescope RH operates has a "mostly environmentally isolated" control room, where the operator can monitor the telescope in nearly complete comfort. Many of the USAF's systems on Maui are even more environmentally isolated. We also define operator environment to include items such as physical security and access to the rooms, equipment, and machinery, travel distance to the observing site, and similar factors.

As always, weather is a factor for successful observing and data collection. Some sites struggle with nearly perpetually cloudy conditions, sometimes for large portions of the year. Other sites overcome these issues because of their high-altitude location, but may struggle with extreme winds or unsafe operator travel conditions. The astute operator should be able, over time and with experience, to gain an 
understanding of the quality of the data likely to be obtained in a given session based on atmospheric conditions and seasonal weather patterns.

Finally, the data collected during an observing session must be appropriately saved, cleaned, configured, and distributed. This is not as problematic if the observatory is internet-connected, but since some observatories are in remote areas (in order to avoid light pollution and adverse atmospheric effects) or are impacted by policy decisions, internet availability can be questionable or unavailable entirely. This is particularly troublesome for device drivers and software packages that have small "installers" and "updaters" that expect to download the remainder of the software when they are executed. For military installations, internet availability may be out of the question altogether, making certain forms of software installation and data distribution particularly challenging.

Clearly, for every observatory, the exact details of the system will differ. This abstract representation is sufficient to highlight and discuss the similarities and differences between the partners involved in the GOLDS-II study.

\subsubsection{Observatory human-in-the-loop}

Now that we have examined the general observatory system, let us look more in-depth at how humans interact with the system - especially from the perspective of the $\mathrm{RH}$ site. Unlike the general representation, which treats all components as interacting portions of a single system, Figure 9 depicts the system viewed from the perspective of the operator and the attendant on the nights when data collection occurs. This view of the system is particularly important as we consider the implications of human factors decisions impacting the success of the data collection effort. Notice how interactions with the system and levels of experience needed may differ, based on whether the actor is an operator or an attendant. 


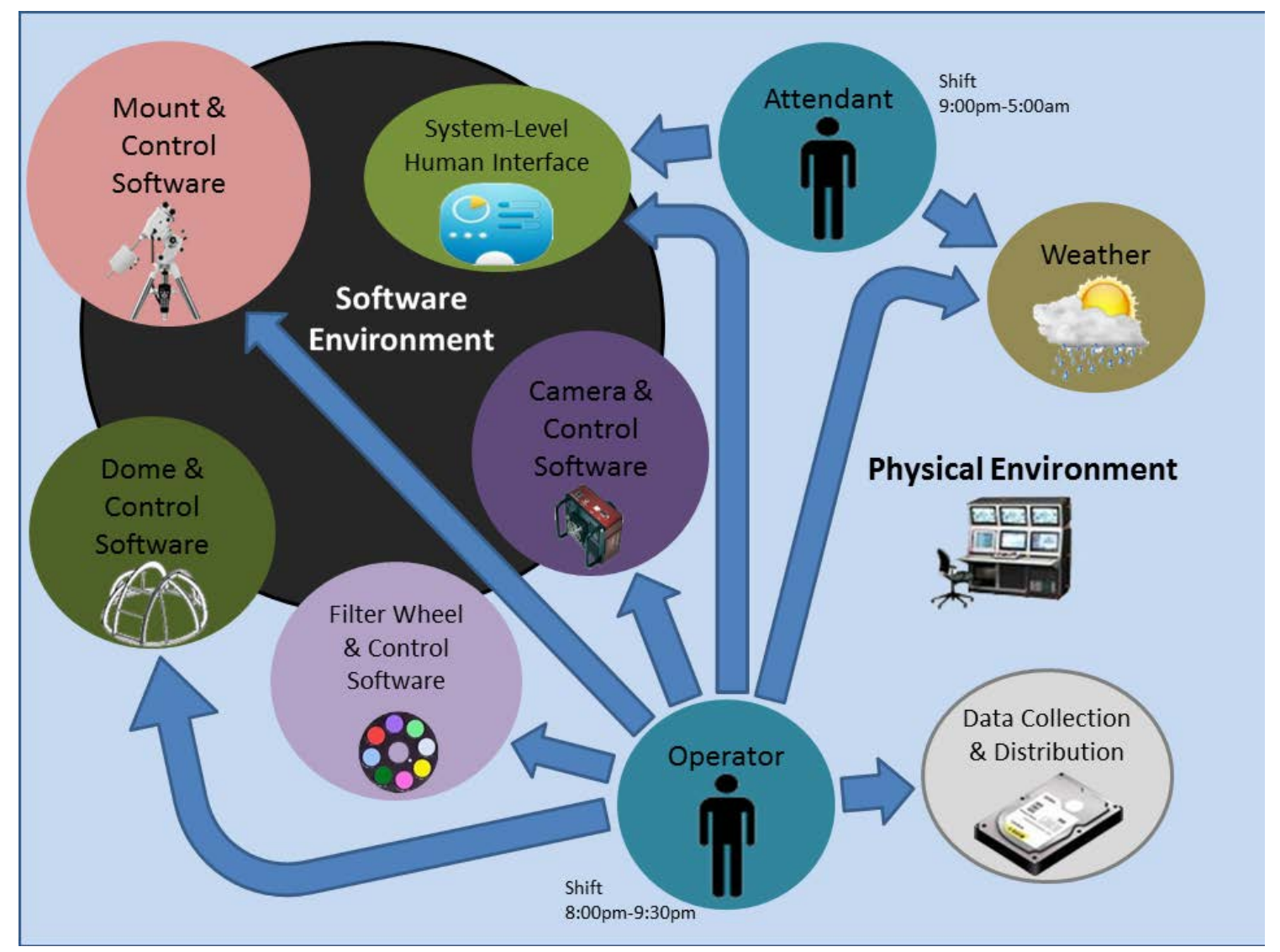

Figure 12: Software and physical interactions of the operators and attendants at RH site

As stated earlier, we defined an operator as an individual who is equipped to configure the system for a night's observing schedule, starting from a shutdown, standby, or similar idle state. The operator's role is to power and initiate the system, leaving it in a steady state for data collection. This requires an operator to be familiar with all of the observatory's component parts, working from a mental model of how every piece of hardware and software (including the control human interface) interacts toward meeting the overarching collection goals. The operator has an explicit view and knowledge of the dome, mount, camera, filters, and their respective controllers and communication protocols. Thus, the operator is generally able to quickly troubleshoot unexpected issues when the system appears to be misbehaving.

A data collection session is performed by an attendant (who may have also been playing the role of operator). An attendant has the knowledge and skills necessary for capturing the anticipated imagery, and interacts with the system GUI as needed to perform the required imagery and data collection tasks. Although the figure suggests the attendant interacts solely through the system GUI, which involves interacting with all of the subsystems, the GOLDS study (in RH's case) would only require an attendant to regularly interact with the camera controller and the associated filter control software. At the end of the session, an attendant would perform additional limited duties for shutting down the observatory or placing the observatory into a "safe mode" using a combination of the software GUI and specific hardware interfaces.

Regardless of their role, all personnel must deal with issues related to weather, securing the session's data, and interacting with the general environment. Note that for most operators and attendants, there is 
practically no interaction with (or even knowledge of!) the telescope's optical design once a decision has been made to participate in data collection sessions, since a telescope's optical design is generally static.

\subsubsection{Multiple site human-centric issues}

As with similar processes, there are several places where the human injects expertise into the system (see Figure 10). These interactions not only provide controls to the overall system, but also supply the context within which the results are evaluated. This makes the human user both a producer and consumer of the system's products. Note that the human is an active participant in each process listed in Figure 10, particularly after the collection components of the system.

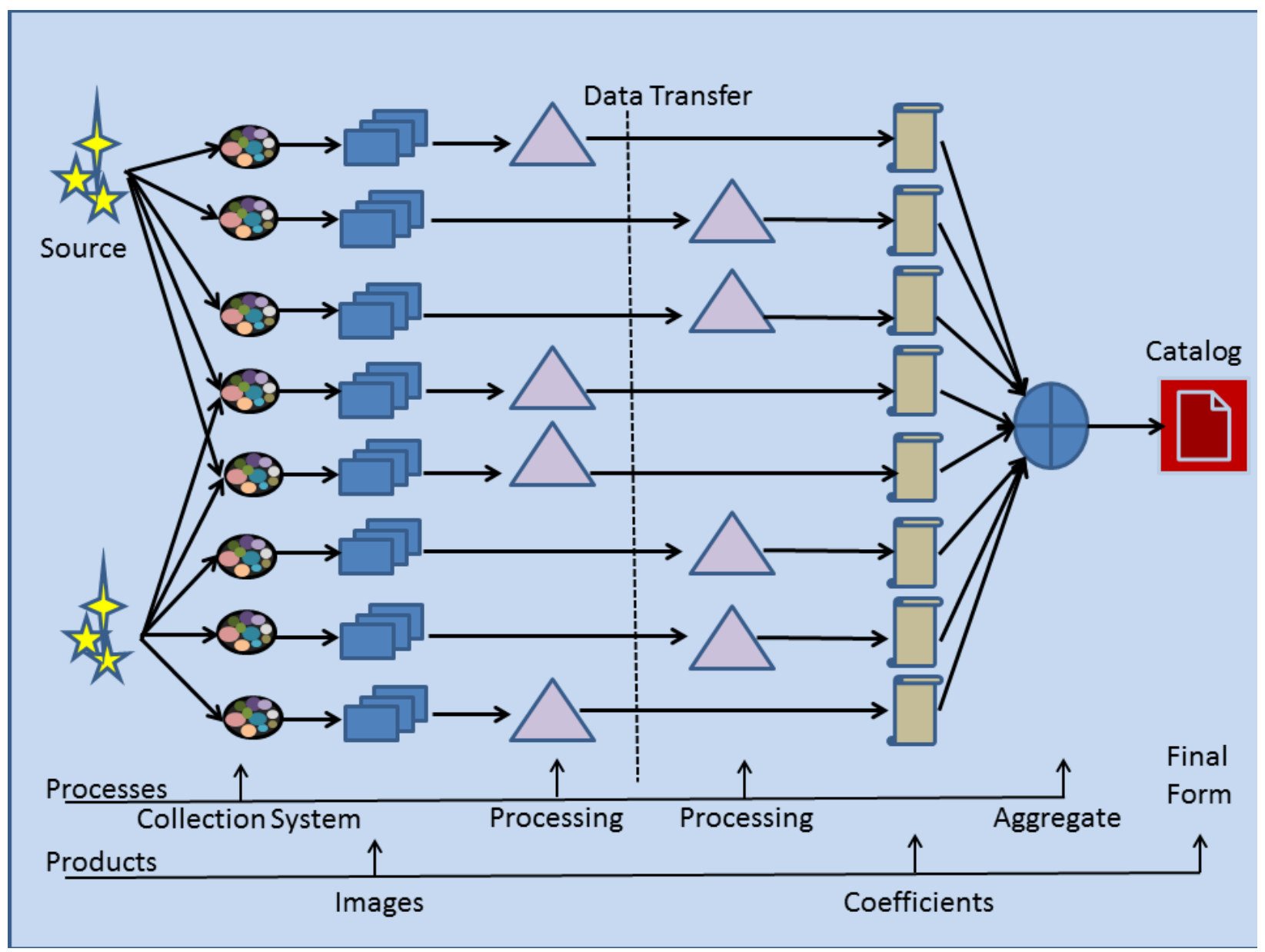

Figure 13: Processes and products for GOLDS-II Collection and Analysis.

Part of the responsibility of good human factors designs includes determining when, where, and how to automate system behavior, and how to optimize the interfaces and interactions between the machine and human components of the system. For observatory systems - and, indeed, for many similar types of interactive machines - a common automation technique involves relegating to the system (hardware and software) those tasks for which computerized and mechanical systems excel: tasks requiring repetition, extreme precision, recognition of well-defined patterns, massive data search, vigilance in well-specified environments, interaction in dangerous or uncomfortable environments, or the application of brute

Distribution A: Approved for public release: distribution is unlimited. 88AB/PA Cleared 11/06/2014: 88ABW-2014-5151. 
strength. Some of these tasks can be managed through careful design of system and subsystem protocols, while others may still rely on regular human interaction and interfaces.

According to the items just enumerated, the GOLDS study is an "easy" task for an observatory system in several respects. Observatories are configured to locate and track remote objects in space (by definition), so locating GEO satellites in known locations is practically trivial: once the objects are located and brought into the field of view, the tracking can be disabled in most cases. This limits a human operator's necessary interactions to those activities required to initialize the observatory system and prepare to capture the desired satellite imagery. (We've deliberately ignored the collection of calibration imagery, which is well within the scope of regular observatory activities.)

For $\mathrm{RH}$, the attendants have the more difficult task, since they are neither astronomers nor regular observatory equipment operators. In their case, two training sessions are provided to familiarize them with the operational procedures and the gear. After an overlapping period of time with the evening's operator, the attendant is left "babysitting" the equipment for the remainder of the session. Their responsibilities involve maintaining situational awareness throughout the evening to keep the satellite images within acceptable parameters, to keep an eye on the weather conditions, and ultimately guide the system into a safe state at the end of their session, which typically happens early in the morning, following a full night of observing.

\subsection{Shift issues}

Given the limited duration of the GOLDS study, few long-term nocturnal issues arose. However, in one case at the RH site, an operator missed his shift when he was scheduled on consecutive nights. In prolonged nighttime "graveyard" shift operations, however, people often encounter issues with getting to sleep, staying asleep, waking, and drowsiness [Drake, et al, 2004]. The issues with circadian desychronization, often resulting from jet lag and shift work, have been attributed to several aircraft accidents as well as mishaps in other work environments such as law enforcement health care and other nighttime occupations. [Ragan, 2007] With a larger pool of operators and attendees, it is much easier to schedule shifts that avoid circadian issues and issues in daily lives. Granted this is not always possible, especially for many observatories with small staffs.

Another issue of shift work that should be considered is shift changes. When there is a division of labor or shifts, some overlapping time is beneficial, if not critical, to ensure the next shift is updated and ready to take over [Miller, 2013]. For GOLDS in $\mathrm{RH}$, it was necessary to pass along information about the targets of the collection, issues encountered in the startup such as weather conditions and visibility, and modifications to standard practices. For example, a new form was introduced to keep track of changes to the camera exposure early in the study, so it was necessary to explain the form to the attendants.

\subsection{Information sharing}

Some of the teams involved in GOLDS had not previously worked with some or all of the other GOLDS teams and thus had not built up close working relationships. This fact, plus the differences in locations and time zones, made it difficult to know what was going on in the other sites. Some sort of chat or verbal communication channel could possibly enable synergistic use of human resources. For example, a novice attendant may have questions in the middle of the night which could be answered by one of the more experienced teams in another location. For GOLDS, a central coordinator was available to respond to questions during business hours, but the teams at each location operated independently. 
Related issues can also exist within the team at a specific site, especially if the team members don't generally interact with one-another on a regular basis. For example, although each operator volunteered to be a POC for any problem an attendant might have at $\mathrm{RH}$ (and one operator was on stand-by for the entire study), attendants were initially hesitant to call in the middle of the collection (the early hours of the morning) if issues would arise that they could not face themselves. (The RH approach that addressed this issue: the operator says "Call me. If you weren't here, I'd be here all night anyway, so I can certainly help with a few questions you might have over the telephone!") Note: a telephone may not be available at all sites, so it may be necessary to ensure an alternate communication method is available.

In the survey, when asked what changes would be beneficial to the effort, one team felt it would be helpful to know when the expected glint times would be. With this information, adjustments could then be made manually to the exposure times within the tasking file. Only one exposure time could be specified when creating the tasking file but adjustments could be manually made to the tasking file where needed.

\subsection{Physical ergonomics}

There were a few noteworthy ergonomic issues at the $\mathrm{RH}$ site mostly during startup and shutdown. Similar issues seem to exist at other sites as well. For example, we found it necessary to operate the control room computer on the lift in addition to the control room floor. Therefore we needed a second monitor and keyboard on the lift which was connected via a USB cable. During startup it was necessary to plug in this USB cable and unplugged it during shutdown. This plug was reasonably accessible for all of RH's telescope workers but it underscores the unconventional steps that needed to be taken. In many cases, it simply makes more sense to trade a little inconvenience rather than spend resources eliminating some unconventional steps.

Dome control could also be an issue, especially for smaller domes. Unless they are fully automated (including opening and closing), domes sometimes require physical agility and strength to operate. At the $\mathrm{RH}$ site, once the telescope was lifted into the dome there was little room to move around, thus it was necessary to rotate the dome to make it accessible for manual opening and closing. In addition, the dome shutters required some upper body strength to move it and required stepping on a small step ladder to access the shutters and locking pin.

\subsection{Safety}

Although telescope operations are generally safe overall, there are some potential hazards that should be considered including the (remote) possibility of telescope slipping in the mount; issues maneuvering the telescope and dome; and, in the case of the RH site, scissor lift safety issues.

Although considered small by professional astronomy standards, Raven-class telescopes are often quite heavy and unwieldy. During installation, lifting RH's 104 pound optical tube assembly (i.e., telescope) onto the mount and securing it takes three or more people to do safely. The U.S. government has no standards on weight lifting but the National Institute for Occupational Safety and Health (NIOSH) has a model which helps predict the risk of injury based on the weight being lifted and other factors [8]. The model is based on medical research about compressive forces that can cause injuries to back bones and ligaments.

All of the GOLDS telescopes employed some level of automation and little, if any, physical interaction was required with the telescope except during startup and shut down. During startup at the RH site, the operator would step onto the lowered scissor-lift platform then raise it about 9 feet up to the dome, where the dome door could be opened. The dome door could require a fair amount of effort to fully open and close thus could potentially result in minor accidents if not careful. The operator needed to be cognizant 
of the potential for pinched fingers and would usually need to step onto a step ladder in order to get better position to open or close the door.

The RH scissor-lift itself, while safe overall, required workers to be conscious of possible hazards. If riding on the raising platform, it was important to remain entirely within the lift railing to avoid significant pinch points. Low lighting could be an issue when the lift was fully raised, because there was no installed lighting system on the platform. Thus a small, battery-operated LED portable light was clamped to the computer mount which provided an adequate amount of light in the dome area where work was performed.

Most of this information was documented in RH's posted safety sheet, and included in operator and attendant training sessions. 


\section{Recommendations}

This section summarizes recommendations based on the results of surveys and experience gained during the recent GOLDS study. For this section, we will use the term "operator" to include the roles of both the operator and attendant.

\section{General}

1) The human-machine interfaces should be designed and implemented specifically to support the work requirements [Scott, et al, 2003]. In other words, what may be an ideal setup for one site may not be optimal for the mission of another site.

2) There are several noteworthy commonalities. For example, all GOLDS sites employed roughly halfmeter telescopes, with some degree of human-in-the-loop needed for setup. Setup procedures and operational safety concerns were relevant throughout, and must be fully documented.

3) During the initial specification of the observatory's hardware and software systems, it is advisable to research best practices by meeting with other observatory operators and performing literature searches. There are many components needed for telescope systems and not all components work well with each other. Understanding the tradeoffs of Component A or Component B ahead of time can save time down the road.

Environment - The operator environment is a key element, especially when the site is not fully automated.

4) Operators should dress appropriately for the working environment. If personnel expect to be exposed to the elements, appropriate clothing will improve comfort and help the operator stay focused on their work.

5) Nighttime alertness and performance can be significantly enhanced with bright ambient light [Campbell \& Dawson, 1990]. That said, some resent research indicates that over-illumination can have diminishing returns - especially on worker announce and health [Boyce \& Boyce, 2003]. Even more recent research indicates benefits of blue-enriched lighting on productivity and circadian phase issues [Najjar, et al, 2014]. (Of course, ensure that the lighting won't interfere with data collection.)

\section{Personnel}

6) Ensure personnel are adequately trained on all operational and safety procedures they will be expected to perform. Documented safety procedures must be visible and accessible.

7) Clearly post emergency contact information where the work is being performed. This is particularly important given that much of this work happens after hours.

8) When data collection is a high priority, make sure personnel do not hesitate to call for help when required. Clearly communicate a no-retribution policy for nighttime contacts.

9) The operator should be mentally engaged (but not overloaded), which will discourage fatigue and promote high work quality. Numerous related studies on vigilance talk about the relationship between vigilance, workload, and stress, and highlight the importance of rest periods as a mechanism to improving performance. [Scerbo, 1998]

10) Prepare high-quality training and operational materials that are tailored to the site (not just the generic manuals that come with some systems), and ensure these are readily available. Incorporate the sitespecific materials into operator training.

11) Ensure that personnel know what to expect during the course of their involvement in the study. Physically walk through the facility and discuss the procedure (using the training documentation as a guide). Point out key decision points and contingencies. 


\section{Staffing}

12) For sites where only a single person is available to operate the system, it is advisable to revise their daytime work schedule to accommodate this task, and make it their only responsibility. This will minimize fatigue and errors during data collection and equipment operation.

13) For sites with multiple operators, if this is not their sole responsibility, be prepared to offer 0.5-1 day before or after their shift as compensated time, and to allow for adequate rest and sleep. (Some people have a very difficult time dynamically rearranging their sleep schedule.) [Warm et al, 2007]

14) Avoid scheduling overnight operators on adjacent nights if overnight telescope operations are not their sole responsibility. They may not be well-rested, and may miss shifts, perform poorly, accidentally misuse equipment, or endanger themselves and others.

15) Encourage volunteers to assist when possible, especially for tasks requiring limited training. Many people are eager to use telescopes in any capacity. This allows others to participate and strengthens awareness of related research. The participation of volunteers also fosters team identity and a sense of ownership of (and responsibility for) the research.

16) If multiple personnel are staffing a single session, rotate them through specific work tasks in order to reduce boredom and fatigue. [Scerbo, 1998]

\section{Human-system interface controls}

17) Even for highly automated systems, advanced users should be able to manually perform certain tasks as situations dictate.

18) Assuming that operators across multiple sites become familiar with common human interface controls ("The Sky X Pro" observatory software, or MaxImDL camera software, for example):

a) Training time may be reduced when operators use telescopes at different (non-home) sites.

b) Operators can solicit, receive, and actively use advice from personnel at other sites with similar software interfaces or hardware configurations.

c) Recognize that each site or sub-site is different, even if the hardware and software are identical. These changes should be clearly documented and briefed.

d) Non-standard equipment isn't generally controlled by these common interfaces. Ensure training and documentation is available.

19) Not all sites use the same control systems. Some are commercial, while others use fully custom systems. Even for customized systems, the terminology is frequently common, and similar functions tend to operate in similar fashions. (Therefore, an operator's knowledge is often transferrable.)

\section{Operation}

20) Automation is a powerful tool for structured and repetitive tasks. While humans bring flexibility, ingenuity, and innovation to job performance, we are also often the source of errors, inaccuracies, and accidents. Before inserting automation technology into a process, consult personnel at other sites on their lessons learned regarding technology insertion, limitations to be aware of, and strengths to leverage.

21) When enlisting new individuals to help in the observatory, assess their strengths and interest before making final decisions on their role. The assessment is often best in the form of synchronous communications like face-to-face or telephone to read body language and voice inflections, but e-mail or other media can also be effective. It is often helpful to allow new personnel to shadow existing 
operators during at least one shift in order to assess their comfort level with an intended role, and to assist with training.

22) Develop procedures that take advantage of technology and timing. For example, check system operation during the day, while repair services are available; collect, save, and analyze images from the night before each morning so changes can be made for the upcoming session if needed. Much of a training regime can also be accomplished during the day.

23) Some systems are capable of collecting data autonomously based on tasking provided the previous evening. Personnel at observatories just getting these features will need to become acquainted with the system's relevant control and scripting languages to ensure the data collector can dynamically adjust exposure time, etc. to prevent an entire evening of collection from being over- or underexposed.

24) It is valuable to perform test runs using similar setups prior to participating in actual data collection efforts. These tests also provide excellent training opportunities, and are good times to validate the operational checklists and related documentation.

\section{$\underline{\text { Logistics }}$}

25) Operators should clearly identify the satellites and features appearing in the images while that data is present and readily available. For example, if images will include two or more satellites, annotate which satellites appear in the image, and where they exist in the imagery (using a readme.txt file, marking a sample image, or making notations in the evening's collection paperwork). It can be tedious to reproduce that information later. (A month after the collect, if some images contain three satellites, it can be time consuming to determine which satellite is which, but it was obvious on the night the data was originally collected - the collection night is the ideal time to capture this information.)

26) Personnel should receive as much training as possible before their scheduled shift. Prepare taskspecific observing documentation as exemplified in Appendix $\mathrm{C}$ - Attendant training material.

27) Although rest periods are important, make sure operators are aware that data collection and telescope operations are their primary responsibilities during their shift. (This especially relates to adjusting the camera parameters to ensure that images do not saturate the camera CCD.) 


\section{Conclusions}

This human factors study provided some useful insights into the human-centric issues of telescope data collections, and formulated recommendations and guidelines to improve processes. This paper may also help sites that are new to such data collection efforts by improving their understanding of the challenges and options available.

The guidelines and conclusions presented were gleaned from surveys of the participants in the recent GOLDS study, as well as from our personal experiences gained during the course of the study. Much of this paper focused on the details of the RH site, since the authors were "hands on" in these operations. As the first significant data collection for this site, some of the lessons learned dealt with our collective inexperience with telescope operations. A significant reliance was placed on the one amateur astronomer in the group to get things running and train others on the tasks that they would perform. Without at least one person with such experience on the team, it would have taken considerably longer to get the operation running.

Despite some similarities, the various sites involved in the GOLDS effort had distinct differences in their setups, manning, and operational procedures. Some sites required overnight manning while others could rely fully on automation. Some personnel performed data collection as their full-time job, while other sites assigned volunteers to cover a few nights during the month. Some sites had controls and personnel located near the telescope they operated while others operated the telescopes remotely. All of these differences can make it difficult to generalize and apply these human factors recommendations. This, however, underscored the need for each organization to have facilities, equipment and procedures that best fit their manpower (how many people are available), personnel (what are their skills), and mission needs. Mission needs include how often will the observatory be used, and for what purposes.

It is our hope that the insights and analyses presented within this report are used to improve the quality of the science being accomplished, while simultaneously improving the conditions and environment within which humans agents must interact. We are, after all, acting both upon the system and within it. 


\section{References}

[1] Air Force Instruction 10-601 (2006) Capabilities-Based Requirements Development - 31 July 2006.

[2] Air Force Research Laboratory Directed Energy Directorate (2012) Air Force Maui Optical \& Supercomputing Site (AMOS) Fact Sheet, http://www.kirtland.af.mil/library/factsheets/factsheet.asp?id=16930, posted 4/30/2012, accessed 6/1/2014.

[3] Boyce, P. and Boyce, B. R. (2003) Human Factors in Lighting, 2nd ed., Taylor \& Francis, London, ISBN 0-7484-0950-5.

[4] Campbell, S. S. \& Dawson, D. (1990). Enhancement of nighttime alertness and performance with bright ambient light. Physiology \& Behavior, 48, 317-320.

[5] Cognion, R. L. (2013) "Observations and Modeling of GEO Satellites at Large Phase Angles", 2013 AMOS Conference Proceedings.

[6] Drake C.L., Roehrs T., Richardson G., Walsh J.K., Roth T., (2004) "Shift work sleep disorder: prevalence and consequences beyond that of symptomatic day workers." Sleep. 2004 Dec 15; 27(8):1453-62. PMID: 15683134.

[7] Fulcoly, D. (2013) "Geostationary Observations with Latitudinal Diversity Simultaneously (GOLDS): Characterizing GEO Spacecraft Pose and Panels Using Photometry", 2013 AMOS Conference Proceedings.

[8] Miller, J.C. (2013). "Fundamentals of Shiftwork Scheduling, 3rd Edition: Fixing Stupid". Smashwords.

[9] Najjar, R.P., Wolf, L., Taillard, J., Schlangen, L.J.M., Salam, A., Cajochen, C., Gronfier, C. (2014) Chronic Artificial Blue-Enriched White Light Is an Effective Countermeasure to Delayed Circadian Phase and Neurobehavioral Decrements, DOI: 10.1371/journal.pone.0102827.

[10] Ragan, B. (2007) “Lag: A Look at Circadian Desynchronization”, ISBN 978-1-4357-0221-9.

[11] Scerbo, M. (1998) "What's So Boring About Vigilance." In Viewing Psychology As a Whole, pp. 145-166. Edited by Hoffman, R., Sherrick, M, and Warm, J.

[12] Scott, R., Roth, E., Deutsch, S., Eggleston, R., Kuper, S. and Whitacker, R. (2003) WorkCentered Support Systems: A Human-Centric Approach to Intelligent System Design, AFRLHE-WP-TR-2004-0043.

[13] Shattuck III, J. L., Schmidt, V. A., Campbell, P. (2012) "Installation and Adjustment Procedure for the Portable Pier and PlaneWave CDK-17 Telescope”, AFRL-RH-WP-TR-2012-0122.

[14] Software Bisque, (2013) "TheSkyX Professional and Serious Astronomer Edition User Guide", available for download at http://www.bisque.com/.

[15] The National Institute for Occupational Safety and Health (NIOSH), (1994) "Applications Manual for the Revised NIOSH Lifting Equation", DHHS (NIOSH) Publication Number 94-110. 
[16] Warm, J., Matthews, G., and Finomore, V. (2007) "Vigilance, Workload, and Stress." In Performance Under Stress, pp. 116-141. Edited by Hankock, P. and Szalma, J. 


\section{Acknowledgements}

We would like thank those at other GOLDS sites - AFRL Maui, AFRL Kirtland (Albuquerque), Air Force Academy (Colorado Springs), and TASC (Washington, DC area) - for taking the time to fill out surveys and do interviews to help in this evaluation. A big thanks to Jeremy Murray-Krezan at AFRL/RVBYC for allowing us to piggy-back on the GOLDS study which he lead. Also big thanks to our volunteer telescope attendants who managed to stay awake and do a professional job on every one of their overnight shifts: John McIntire, 1Lt Michael "Trent" Schill, Dr. Gina Thomas, Dr. Mike Vidulich, and research student operator Sarah Allison.

Finally, we want to give a very special acknowledgement to our late colleague and friend Judson "Skip" Shattuck, III. Skip unexpectedly passed away on December 4,2013 at the age of 53 - about a month after the GOLDS experiment concluded. Skip was a key player in the Human Effectiveness $(\mathrm{RH})$ telescope installation and the engineering involved to motorize and automate the dome. He was one of four people at the RH site who could fully operate the telescope. Skip was always concerned with doing the job correctly and the safety of those he worked with. He is greatly missed.

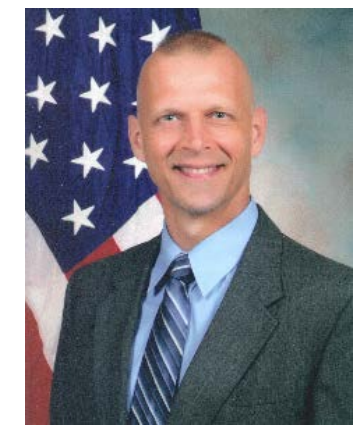

Judson "Skip" Shattuck, III 


\title{
Appendix A - Site startup and shutdown procedures \\ Telescope System \\ Startup and Shutdown Procedures \\ for GOLDS-II Data Collection
}

\author{
Updated 28 Oct 2013
}

!!! CHECK WEATHER BEFORE YOU BEGIN \& REGULARLY DURING COLLECTION !!!

\section{STARTUP (Operator)}

1. Ensure the scope \& pump power disconnect switches are off, \& unplug external monitor USB

2. Reboot the Control PC and log in

3. Energize the scope and pump power disconnect switches after the PC has booted

4. Turn on air compressor

5. Plug in the external monitor USB

6. Make sure the Dome Controller power is on (wall switch is near gray box)

7. Visually inspect cabling to ensure OTA and camera cables can move freely

8. If the OTA is not in its park position, use the hand controller to approximately level the OTA

9. Start the MaxImDL application using the desktop shortcut
(a) Start Observatory dialog,
(b) Select "Connect All" to connect to STI (telescope control) and PWI (focuser)
(c) Close the Observatory dialog

10. Start TheSkyX Pro application using the desktop shortcut
(a) Select the Telescope tab at the left of the screen,
(b) Select "Look Up" icon on ribbon menu at the top of the application screen

11. Ride the lift to the top (adhere to posted Observatory Safety Tips)

12. Check dome tire air pressure, inflate to $50 \mathrm{PSI}$ if required.

13. Start the Dome Control application
(a) Select "Connect Dome" button at the top of the application screen
(b) Rotate dome using the buttons at the bottom of the application until the shutter is accessible

14. Open the dome shutter (manually) by removing the pin and pulling the handles apart

(a) Store the pin in the dome apron

15. Using the Dome Control application:

Use the buttons at the bottom to center the dome shutter directly in front of the OTA

16. Ride the lift down; do not lower the lift completely in order to avoid jarring the telescope

17. Turn off air compressor

18. Using STI (the telescope control application):
(a) Select "Home" from the Command pulldown in the center of the screen
(b) Select "Initialize Scope Using Homing Switches" in "Homing Operations Window" dlg
(c) Wait for initialization to complete ("Idle" status on both axes), and press dialog's "OK"
(d) Select "Start" in the center of the STI screen to begin telescope tracking.

19. Disconnect the remote monitor USB

20. Using TheSkyX Pro: select "Connect Telescope" from Startup menu in Telescope tab

21. Using the Dome Control application: Select "Connect Scope"; Select "Sync"; Select "Autotrack"

(NOTE: The dome may require additional positioning adjustments to ensure accuracy.)

22. Use the yellow hand controller to raise the lift to the top (with no person on the lift) 


\section{FILE, CAMERA, \& MODEL INITIALIZATION FOR DATA COLLECTION (Operator)}

\section{Data files, Load TLEs, and Camera Init. \& Focus}

1. Using File Manager:

(a) Ensure any of last night's *.FIT files in
Desktopllmages are moved to
Desktopllmagesl2013MMDD GOLDS2

(b) Create a directory for tonight's data files: DesktoplImages|2013MMDD GOLDS2

2. Using TheSkyX Pro:
(a) Select menu item: Input $\rightarrow$ Satellites
(b) Select "Remove All" to clear satellites
(c) Select "Import from File" and open file DesktopITLE2013MMDDIgeo.txt
(d) Close the Satellites dialog

3. Using MaxImDL's Camera Control dialog (icon to the left of Observatory icon):
(a) Setup tab, select Connect button (connects camera \#1, filter wheel, and guide camera \#2)
(b) Select Coolers On button (toward mid-right in Setup tab)

4. Set focus using the PWI application (focuser):
(a) Select "Start AF"
(b) Wait for autofocus images to be taken (there are 5) and processed
(c) It is generally a good idea to use MaxImDL to take one more photo, just to make sure you concur with the autofocus setup: select the "Focus" preset \& set values to use.

(d) Minimize PWI

\section{Mount Model Correction}

1. Using TheSkyX Pro, select a bright star (Deneb, Altair...)

(a) left-click the star, right-click for a popup menu, select "Slew",

(b) press dialog's OK button to confirm the slew

2. Set exposure settings in MaxImDL's Camera Control dialog's Expose tab to reasonable values

3. Repeat as required: (using MaxImDL for camera control app \& STI for telescope control)

(a) Using MaxImDL's Camera Control dialog's Expose tab: select Start to take photo

(b) Proceed to (d) if the bright star is centered

(c) If bright star is not centered, use STI to move the telescope:

i. Set Rate to Pan (med) or Slew (fast)

ii. Use cursor keys (very briefly!) to move telescope in very small increments

4. Use TheSkyX Pro to save model correction for session:

(a) NOTE: make sure the desired star is selected in TheSkyX Pro (red bulls-eye) before Sync!

(b) From the Telescope tab at the left, use the Startup menu and select Synchronize

(c) Press the "Sync" button on the dialog, and say "Yes" to confirm the sync.

(d) Be patient! This will take a few moments.

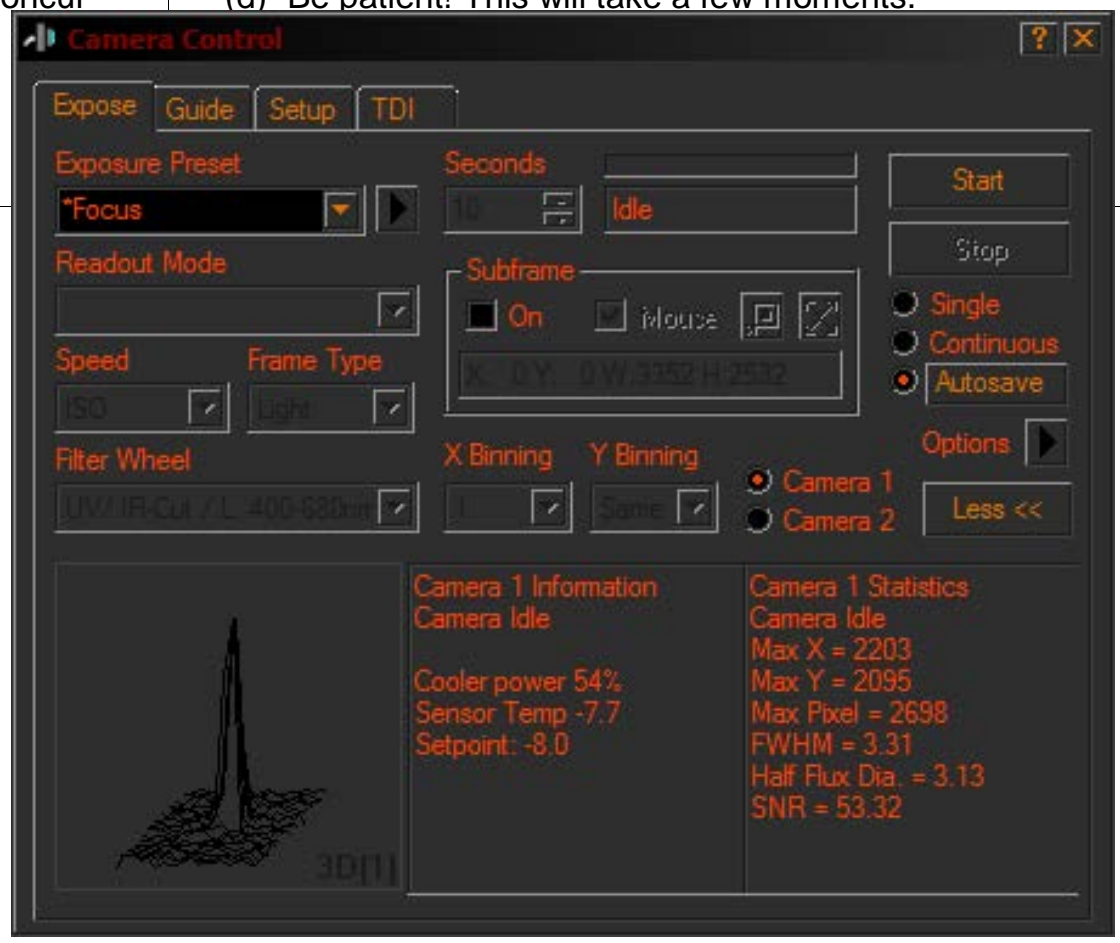

35

Distribution A: Approved for public release: distribution is unlimited. 88AB/PA Cleared 11/06/2014: 88ABW-2014-5151. 


\section{CALIBRATION FOR DATA COLLECTION (Operator)}

Actual collection of satellite data should occur between 9pm and 5am (nominally). GOLDS II PM's have asked us to run about $5-10 \%$ of our time each night for three (3) of the calibration settings to characterize the night's observations. That amounts to about 30 minutes total (10 minutes per setting). If an operator begins setup near $8 \mathrm{pm}$ and is running calibration by 8:30pm, data collection can start shortly after $9 \mathrm{pm}$.

Calibration stars: - Collect 10 minutes of data on each set of calstars: SA109 \& SA1105

1. Use TheSkyX Pro to slew to the calibration star

2. Use MaxImDL's Expose tab on the Camera Control dialog

a. Take a single photo, 20 seconds or so, binning $2 \times 2$, using the UV/IR Cut filter

b. Compare the image to confirm the selected setting is in the field of view; adjust using STI if necessary. (Note: telescope will be in tracking mode for these operations)

c. Take a single photo using the Red filter (exposure @ 25 seconds)

d. Adjust exposure and repeat until max pixel for Red is $\sim 10 \mathrm{~K}$

e. Take a single photo using the Blue filter (exposure @ 30 seconds)

f. Adjust exposure and repeat until max pixel for Blue is $\sim 10 \mathrm{~K}$

g. Set Autosave options (dialog will appear) to capture Red (suffix=R) and Blue (suffix=B), exposure as discovered in previous steps for respective filters, Binning 2, Repeat 9999. Select "Ok" in dlg.

h. Press Start in the Camera Control dialog, and allow the system to take 10 minutes of data.

\section{Set up for Satellites:}

1. Choose Setting E1-E4 based on the night's observing schedule. For makeup days, select a setting that was not collected recently, or has not been collected as frequently as the others.

2. Using TheSkyX Pro:

a. Slew to the desired Setting.

b. Zoom and reposition until scope (yellow bulls-eye) is in the "camera view" purple square

c. Play the "geo satellite game" to center the scope between both satellites for the Setting

i. Be ready to turn tracking off ("Stop" in STI)

ii. Remember, using STI's RA/DEC buttons turns tracking back on!

iii. You will be moving between TheSkyX Pro, STI, and MaxImDL frequently.

iv. Use the Dome Control application to turn dome Autotracking off

3. Use MaxImDL's Expose tab on the Camera Control dialog

a. Take a single photo, 20 seconds or so, binning $2 \times 2$, using the UV/IR Cut filter

b. Examine the image to confirm both satellites of the selected Setting are in the field of view; adjust using STI if necessary. (Telescope tracking must be off for these operations)

c. Image should contain single dots (satellites) in good focus, "star trail" streaks are possible

d. Take a single photo using the Red filter (exposure @ 5 seconds)

e. Adjust exposure and repeat until max pixel for Red is in the range (2000-10000)

i. Use the Information dialog (icon to the left of the toolbar's Camera Control icon)

ii. The Area mode (selectable from the pulldown menu) allows a box to be drawn around an area of an image.

iii. After boxing the satellite, use the max pixel value in this dialog to guide exposure

f. Take a single photo using the Blue filter (exposure @ 8 seconds)

g. Adjust exposure and repeat until max pixel for Blue is the range (2000-10000)

h. Set Autosave options (dialog will appear) to capture Red (suffix=R) and Blue (suffix=B), exposure as discovered in previous steps for respective filters, Binning 2, Repeat 9999. Select "Ok" in dlg.

i. Press Start in the Camera Control dialog

j. At this point, the system is in steady-state for the evening. 


\section{COLLECTING DATA FOR DATASET E1 -E4 (Attendant)}

\section{Steady-State operation (Attendant)}

1. CHECK WEATHER REGULARLY! Immediately execute Shutdown Procedure if the weather becomes uncooperative (excessively overcast or includes precipitation in any form)

2. During the data collection:

(a) Imagery will be captured and saved continuously, \& displayed on the monitor in real time

(b) The telescope will change filters automatically

3. If satellites move out of the image, contact Vince (or designated operator) to make adjustments

4. Check the max pixel values of satellites using MaxImDL's Information dialog (icon to the left of the Camera Control icon in the toolbar):

(a) Use the Area mode (selectable from the pulldown menu) to draw a box around each satellite

(b) The status of the selected area is displayed in real time in the dialog

(c) The maximum pixel value should remain in the range $(2000,20000)$

5. Adjust Exposure as needed to maintain maximum pixel values

(a) Press Stop in the Camera Control dialog's Expose tab to stop data collection

(b) Press the Autosave button \& change the desired Exposure value in the Autosave Setup dialog

(c) Press OK to save the value and dismiss the dialog.

(d) Press Start in the Camera Control dialog's Expose tab to continue collecting imagery

6. Select and use a Shutdown Procedure when the shift is over

7. Fill in the log sheet on the clipboard

8. Make sure the catwalk door is locked before leaving for the evening

9. Send a short email to Vince (vince@vincentive.org) to include:

Setting, start and stop time of data collection, any comments. Please include reason why data collection was halted (end of shift, overcast skies, etc.)

10. Please do not use the telescope Control PC for anything except for data collection and telescope control!

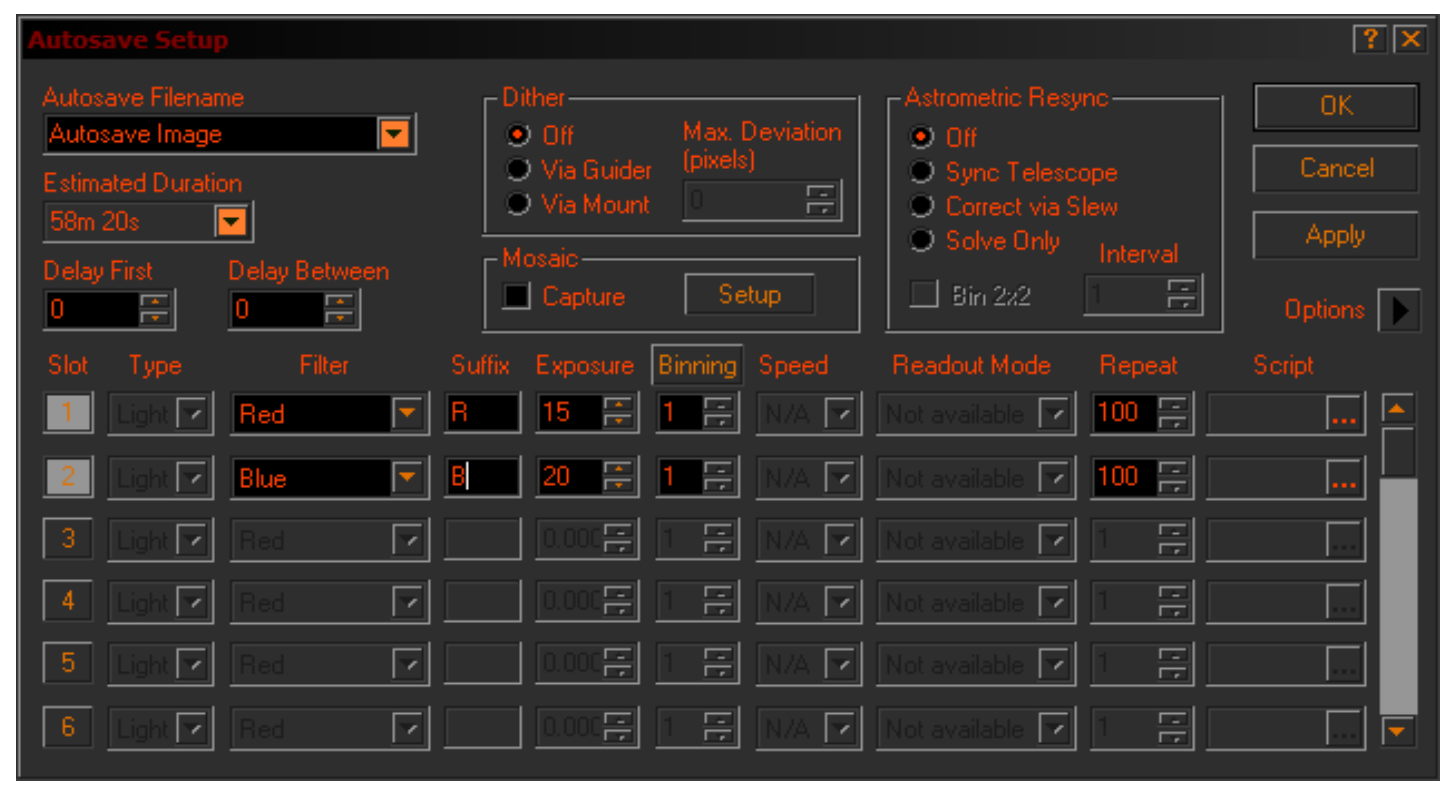

37

Distribution A: Approved for public release: distribution is unlimited. 88AB/PA Cleared 11/06/2014: 88ABW-2014-5151. 


\section{SYSTEM SHUTDOWN}

(Attendant: Select either Brief or Complete Shutdown method)

\section{Brief Shutdown (B): turn off camera coolers; park telescope; close dome}

1. Using MaxImDL (Camera Control dialog):

(a) Select "Stop" from the Expose tab to halt data collection.

(b) Select the Setup tab. Select "Cooler Off" and select "Disconnect" and close the dialog

2. Lower the platform until you would be comfortable stepping onto the platform;

(a) use the yellow pendant on the hydraulic compressor

(b) Don't completely lower the platform using the pendant, this will jar the telescope!

3. Use the telescope hand controller to lower the OTA to a horizontal orientation

4. Connect the remote monitor USB plug (the cable with the orange post-it on the plug)

5. Get onto the lift, and raise the lift completely

6. Secure the Dome:

(a) Use the Dome Control application to rotate the dome shutter for access

(b) Close the shutters manually (be forceful)

(c) IMPORTANT: insert the pin to secure the shutters

7. Gently fully lower the platform - Use the green release lever to control the descent rate

8. Unplug the remote USB monitor (the cable with the orange post-it on the plug)

9. Turn off both wall power boxes; Leave PC logged in.

10. Lock catwalk door 


\section{Complete Shutdown (C): everything will be powered down}

1. Using MaxImDL (camera control):

(a) Select "Stop" from the Expose tab to halt data collection.

(b) Select the Setup tab. Select "Cooler Off" and select "Disconnect" and close the dialog

(c) Observatory Control dialog -Select "Disconnect All"

(d) Close the application

2. Using PWI (focuser): Close the application (it will disconnect automatically)

3. Use the Dome Controller application and de-select "Connect Telescope"

4. TheSkyX Pro:

(e) Select the Telescope tab at the left

(f) Select the Shutdown menu, and choose "Disconnect Telescope"

(g) Close the application

5. Lower the platform until you are comfortable stepping onto the platform;

(h) Use the yellow pendant on the hydraulic compressor

(i) Don't completely lower the platform using the pendant, this will jar the telescope!

6. Using STI (telescope controller):

(j) Select Park from the Command pulldown menu at the center of the window

(k) WATCH the park operation and be prepared to stop the scope by removing power if it does not stop! It IS possible to run the OTA into the mount!

(I) In order: close the ASCOM application, and close the STI application, if they are running.

7. Connect the remote monitor USB plug

8. Get onto the lift, and raise it completely

9. Secure the Dome:

(m) Use the Dome Control application to rotate the dome shutter for access

(n) Close the shutters manually (be forceful)

(o) IMPORTANT: insert the pin to secure the shutters

(p) De-select "Connect Dome"

(q) Close the application

10. Gently fully lower the platform; Use the green release lever to control the descent rate

11. Unplug the remote USB monitor

12. Turn off both wall-mounted power disconnect boxes

13. Lock catwalk door 


\section{List of RH GOLDS-II Personnel}

Oct 2013

Operators (Full training for telescope operations)

\begin{tabular}{|l|l|l|}
\hline Name & Phone & Email \\
\hline Sarah Allison & $\mathrm{XXX}-\mathrm{XXX}-\mathrm{XXXX}$ & $\mathrm{XXX@XXX}$ \\
\hline Jacob Kern & $\mathrm{XXX}-\mathrm{XXX}-\mathrm{XXXX}$ & $\mathrm{XXX@XXX}$ \\
\hline Vince Schmidt & $\mathrm{XXX}-\mathrm{XXX}-\mathrm{XXXX}$ & $\mathrm{XXX} @ \mathrm{XXX}$ \\
\hline Skip Shattuck & $\mathrm{XXX}-\mathrm{XXX}-\mathrm{XXXX}$ & $\mathrm{XXX} @ \mathrm{XXX}$ \\
\hline
\end{tabular}

\section{Attendants (Trained for data collection)}

\begin{tabular}{|c|c|c|}
\hline Name & Phone & Email \\
\hline John Ianni & $X X X-X X X-X X X X$ & $X X X @ X X X . m i l$ \\
\hline John Mclntire & XXX-XXX-XXXX & XXX@XXX.mil \\
\hline Trent Schill & $X X X-X X X-X X X X$ & $\mathrm{XXX@XXX.mil}$ \\
\hline Gina Thomas & $X X X-X X X-X X X X$ & XXX@XXX.mil \\
\hline Mike Vidulich & $X X X-X X X-X X X X$ & XXX@XXX.mil \\
\hline
\end{tabular}




\section{GOLDS-II Log Sheet}

October 2013 Data Collection

\begin{tabular}{|c|c|c|c|c|c|c|c|c|}
\hline \multirow[b]{2}{*}{ Date } & \multirow{2}{*}{$\begin{array}{r}\text { Target } \\
\text { (E1-E4) }\end{array}$} & \multicolumn{3}{|c|}{ Telescope Operator } & \multicolumn{4}{|c|}{ Research Attendant } \\
\hline & & Name & Arrive & Leave & Name & Start Time & End Time & Shutdown \\
\hline 30 Sep & & & & & & & & B C \\
\hline 1 Oct & & & & & & & & B C \\
\hline 2 Oct & & & & & & & & B C \\
\hline 3 Oct & & & & & & & & B C \\
\hline 4 Oct & & & & & & & & B C \\
\hline 5 Oct & & & & & & & & B C \\
\hline 6 Oct & & & & & & & & B C \\
\hline 7 Oct & & & & & & & & B C \\
\hline 8 Oct & & & & & & & & B C \\
\hline 9 Oct & & & & & & & & B C \\
\hline $10 \mathrm{Oct}$ & & & & & & & & B C \\
\hline 11 Oct & & & & & & & & B C \\
\hline 12 Oct & & & & & & & & B C \\
\hline 13 Oct & & & & & & & & B C \\
\hline 14 Oct & & & & & & & & B C \\
\hline 15 Oct & & & & & & & & B C \\
\hline 16 Oct & & & & & & & & B C \\
\hline 17 Oct & & & & & & & & B C \\
\hline $18 \mathrm{Oct}$ & & & & & & & & B C \\
\hline 19 Oct & & & & & & & & B C \\
\hline 20 Oct & & & & & & & & B C \\
\hline 21 Oct & & & & & & & & B C \\
\hline 22 Oct & & & & & & & & B C \\
\hline 23 Oct & & & & & & & & B C \\
\hline 24 Oct & & & & & & & & B C \\
\hline 25 Oct & & & & & & & & B C \\
\hline 26 Oct & & & & & & & & B C \\
\hline 27 Oct & & & & & & & & B C \\
\hline 28 Oct & & & & & & & & B C \\
\hline 29 Oct & & & & & & & & B C \\
\hline 30 Oct & & & & & & & & B C \\
\hline 31 Oct & & & & & & & & $\mathrm{B} \mathrm{C}$ \\
\hline
\end{tabular}

Vince's Cell \#, call 24 hours if there are any problems:937-XXX-XXXX Email: vince@xXX.org

Distribution A: Approved for public release: distribution is unlimited. 88AB/PA Cleared 11/06/2014: 88ABW-2014-5151. 


\section{Appendix B - Attendant log sheet}

Study: GOLDS 2 Date:

Setting:

\begin{tabular}{|l|l|}
\hline Attendant: & \\
\hline Arrive @ & \\
\hline Shutdown @ & \\
\hline Shutdown Reason & \\
\hline Leave @ & \\
\hline
\end{tabular}

Page:

Sat $2 \rightarrow$

Sat $1 \rightarrow$

\begin{tabular}{|c|c|c|c|c|c|c|c|c|}
\hline Start @ & \multicolumn{3}{|c|}{ Red (goal 2000-20000) } & \multicolumn{3}{|c|}{ Blue (goal 2000-20000) } & \multirow[b]{2}{*}{ Stop @ } & \multirow[b]{2}{*}{ Stop Reason } \\
\hline$(\mathrm{V}=$ check $)$ & Exposure & Sat1 max & Sat2 max & Exposure & Sat1 max & Sat2 max & & \\
\hline \multicolumn{9}{|l|}{$\mathrm{V}$} \\
\hline \multicolumn{9}{|l|}{$\mathrm{V}$} \\
\hline \multicolumn{9}{|l|}{$\mathrm{v}$} \\
\hline \multicolumn{9}{|l|}{$\mathrm{V}$} \\
\hline \multicolumn{9}{|l|}{$\mathrm{v}$} \\
\hline \multicolumn{9}{|l|}{$\mathrm{v}$} \\
\hline \multicolumn{9}{|l|}{$\mathrm{V}$} \\
\hline \multicolumn{9}{|l|}{$\mathrm{V}$} \\
\hline \multicolumn{9}{|l|}{$\mathrm{V}$} \\
\hline \multicolumn{9}{|l|}{$\mathrm{V}$} \\
\hline \multicolumn{9}{|l|}{$\mathrm{V}$} \\
\hline \multicolumn{9}{|l|}{$\mathrm{V}$} \\
\hline \multicolumn{9}{|l|}{$\sqrt{ }$} \\
\hline \multicolumn{9}{|l|}{$\mathrm{V}$} \\
\hline \multicolumn{9}{|l|}{$\mathrm{V}$} \\
\hline \multicolumn{9}{|l|}{$\mathrm{V}$} \\
\hline \multicolumn{9}{|l|}{$\mathrm{v}$} \\
\hline \multicolumn{9}{|l|}{$\mathrm{v}$} \\
\hline \multicolumn{9}{|l|}{$\mathrm{V}$} \\
\hline \multicolumn{9}{|l|}{$\mathrm{v}$} \\
\hline \multicolumn{9}{|l|}{$\mathrm{v}$} \\
\hline \multicolumn{9}{|l|}{$\mathrm{v}$} \\
\hline \multicolumn{9}{|l|}{$\mathrm{v}$} \\
\hline \multicolumn{9}{|l|}{$\frac{v}{v}$} \\
\hline \multicolumn{9}{|l|}{$\mathrm{v}$} \\
\hline $\mathrm{V}$ & & & & & & & & \\
\hline
\end{tabular}

Distribution A: Approved for public release: distribution is unlimited.

88AB/PA Cleared 11/06/2014: 88ABW-2014-5151. 


\section{Appendix C - Attendant training material}

\section{GOLDS II Attendant Training Topics to Cover}

Updated 8 Oct 2013

This short document is intended to be a list of topics an Operator must cover to introduce and qualify an individual as a telescope attendant for the GOLDS II study. This document is not a complete "training guide" that can be read and fully understood by inexperienced personnel.

GOLDS II training for attendant personnel can be accomplished in two (2) parts: daytime physical component training, and software operations. Although the software operations training can also be performed during the day (since these are geosynchronous satellites), it is best to cover the software topics in the evening when the camera images can be used to demonstrate the relevant procedures.

\section{General comments:}

Data collection should occur between 9pm and 5am (nominally). An attendant should plan to arrive for their shift NLT 9pm. This will allow for a good handoff from the Operator to the Attendant.

\section{Daytime Physical Component Training (30 minutes)}

The intent of this portion of the training is to familiarize the attendant with the physical components of the observatory system that will be used during system shutdown and (possibly) under limited circumstances during data collection.

- Introduce telescope system - reflecting telescope, truss design; pier/mount/optical tube assembly (OTA). Special coatings to allow collection from 250-2500nm (visual is 400 700).

- $\quad$ Energize (and point out) wall power disconnect switches.

- Point out Control PC.

- Start Dome Control program and STI

- Show remote monitor USB plug.

- Ride lift to top, new Attendant control motion. Lift operations safety brief.

- Show telescope LED light, clipped near monitor.

- Point out all dome emergency stop switches.

- Dome shutter operations (Op: move, open \& move dome; trainee: move \& close dome) 
- Dome shutter pin storage and installation

- Ride lift to bottom; use lever to carefully lower platform.

- Point out friction dovetails. (Op: home telescope)

- Park OTA using hand controller (show controller, slew speeds; trainee: "park" scope)

- Show OTA emergency stops: controller on pier; wall power; software stop

- Log sheet on clip board. Other materials and phone numbers on cork board.

- Walk through complete shutdown procedure: when collection is finished ...

o Lower lift using yellow pendant (not all of the way to floor)

o Level OTA

o Connect external monitor USB

o Ride lift to the top, rotate and close dome, lower lift; disconnect external monitor USB

o Turn off camera coolers and disconnect camera

o Kill power at the wall switches

\section{Software Operations Training (30 minutes)}

The intent of this portion of the training is to demonstrate the operation of key software components that might be used by the attendant in order to prevent unnecessary degradation of the quality of the data collected by the system.

- Show observing schedule (\& primary/secondary attendants), contact info

- Log sheet location and information

- Check weather regularly (show access key and where to look for weather)

o Note: Emergency shutdown procedures are the same as standard Brief procedures

- Walk through shut down procedures

- Keep lab door open during operations, close when leaving

- PC login

- Only use Control PC for telescope operations

- Show PWI (focuser) software

- Show MaxImDL Camera Control for data collection; Start/Stop and Autosave script settings

- Show MaxImDL "target" icon and where to look/what to expect for max pixel values

- Text or email sitrep to Vince: observing times, Setting, shutdown reason, max observed pixel

- Call Vince for any reason 


\section{Appendix D - Manpower scheduling}

\section{October 2013}

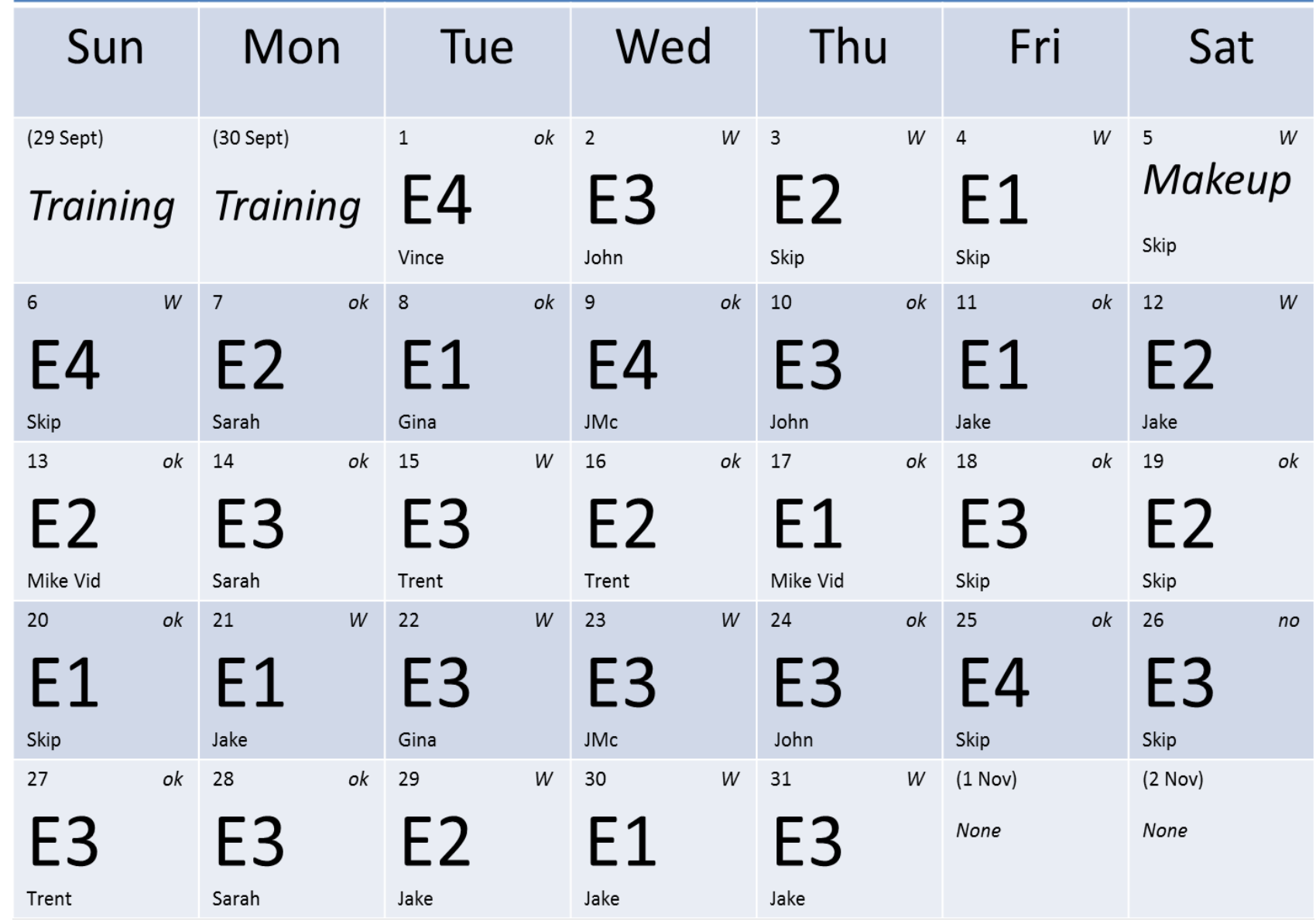




\section{Appendix E - High-level guidance}

\section{RHCV (Dayton) GOLDS II Data Collection (1 Oct - 31 Oct 2013)}

\begin{tabular}{|c|c|c|c|}
\hline Setting & Satellite Name & Satellite \# & Body Type \\
\hline Setting E1 & DirecTV 4S & 26985 & Boeing 601 \\
\hline & DirecTV 9S & 29494 & LSS 1300 \\
\hline Setting E2 & DirecTV 8 & 28659 & LSS 1300 \\
\hline \multirow{2}{*}{ Setting E3 } & SES-1 & 36516 & Star 2 \\
\hline & DirecTV 12 & 36131 & Boeing 702 \\
\hline Setting E4 & DirecTV 10 & 31862 & Boeing 702 \\
\hline & AMC 18 & 29644 & A-2100 \\
\hline
\end{tabular}

- Constant observation of one target setting per night (geo.txt TLE file)

- Filter bands: Johnson Blue \& Red (alter exposure as needed to avoid saturation)

- Email/Text SitReps nightly to Vince: vince@xxX.com / 937-XXX-XXXX

- Include: observation times, setting, etc

- Include: Reason for shutdown ( $w x$, end of shift, etc.)

- Include: Approx. observed max intensity

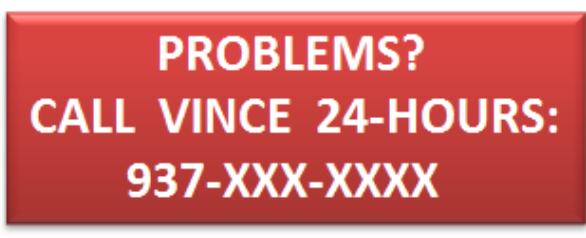




\section{Appendix F - Situation report examples (various sites, not identified)}

Started $\sim 2130$, stopped $\sim 0310$ MDT

USAFA-1 (16-inch) observed East 2 (DTV-8 and SES-1); swapped with USAFA-2 because the 16-inch was able to get both satellites in its FOV.

DTV-8 visible in $\mathrm{R}$ at 3 seconds exposure during shadow period.

SES-1 brightened considerably after shadow, but did not appear to have a sharp glint.

USAFA-2 (Mobile-1) observed East 3 (DTV-10 and DV-12).

Both DTV-10/12 visible in R during shadow period.

Our site collected on East 1 but we had an equipment failure during the automated portion of the run and therefore only have about $15 \mathrm{~min}$ of data.

As mentioned last week, our area was weathered out all last week.

We ended up having a computer glitch last night and got a considerably delayed start. We're asking our folks to be ready to collect data beginning at 2100 . It is sufficiently dark at $8 \mathrm{pm}$ for us to get the calstars started, and we're working on getting started more quickly when possible, but I don't imagine collections starting before $9 \mathrm{pm}$ will be practical yet. Time will tell.

Since we're not crunching numbers for you, I'll need to get imagery transferred to you at some point. It looks like 6GB per evening. Let me know how and when you'd like the images.

Distribution A: Approved for public release: distribution is unlimited. 88AB/PA Cleared 11/06/2014: 88ABW-2014-5151. 


\title{
Appendix G - GOLDS White Paper
}

\section{Proposed Plan for a Simultaneous GEO Observation Campaign}

\author{
Dr. Steve Gregory (Boeing LTS), Dr. Tamara Payne (Applied Optimization) \\ Dr. Jeremy Murray-Krezan, and Lt. Daniel Fulcoly
}

AFRL Space Vehicles Directorate

Prof. Francis Chun

United States Air Force Academy

\section{Introduction:}

AFRL Space Vehicles Directorate has recently organized GEO color photometry data previously collected under a variety of AFRL programs (funding from SMC, DARPA, and AFRL) into a coherent approach to collecting, analyzing, and distributing data and information that have significant impact on SSA characterization for GEO belt objects. Two photometry catalogs have been generated. The first is the GEO Color Photometry Catalog (GCPC), which currently provides more than 112,000 individual brightness determinations for 110 GEO satellites, taken over the last 10 years; this catalog has concentrated on active, stable satellites. The second is the AFRL-RV Deep Space Catalog, which contains approximately 10,000 measures of 60 objects; this catalog has concentrated on observations of deactivated/unstable satellites, rocket bodies, and debris. Together, these catalogs provide important SSA characterization information about the current state and historical changes relevant to a wide range of GEO belt objects. The GCPC is complete for all active, low inclination GEOs over CONUS and Hawaii plus a strong sampling of objects in the $105^{\circ}$ West Longitude Pinch Point. The photometric accuracy of the catalog is estimated at better than 5\% flux, which permits reliable SSA characterization of GEOs.

The military utility of information that has been derived from the above mentioned data sets include the following:

- Current brightness of RSO 
- Historical brightness of RSO

- Algorithmic development that automatically classifies active object by manufacturer/bus type

- Examples of cross tagging resolution

- Examples of anomaly resolution

- Examples of change detections

- Examples of on-orbit aging effects

- Investigation of seasonal illumination changes, including very bright glints.

Under a variety of programs, these kinds of information have been transitioned to the war fighter via the intelligence community and AFSPC.

Furthermore, military utility includes support of photometric characterization of satellites with data from the Space Based Space Surveillance System (SBSS) and the Space Surveillance Telescope (SST). Although both of these Space Surveillance Network sensors have photometric capabilities AFRL data assists in baselining and interpretability.

Recent field campaigns have supported catalog updates and the addition of exquisite SSA characterization data.

\section{GOLDS}

The GEO Observations with Longitudinal Diversity Simultaneously (GOLDS) field campaign was initiated with a general objective of investigating the brightness and colors that GEOs exhibit during the biannual glint seasons, near Equinox. True glints occur when the sun/sensor/GEO geometry is such that the main facets of the satellite (solar panels for 3-axis stabilized satellites) specularly reflect sunlight, toward the sensor (see Figure 1). This special situation often leads to dramatic increases of measured flux, ranging from 10,000 to 100,000,000 times the baseline brightness of $10^{\text {th }}$ to $14^{\text {th }}$ magnitude in visible wavelengths typically received from GEOs. Understanding of glints and near-glinting brightness permits exquisite SSA characterization of RSOs and RSO components. 


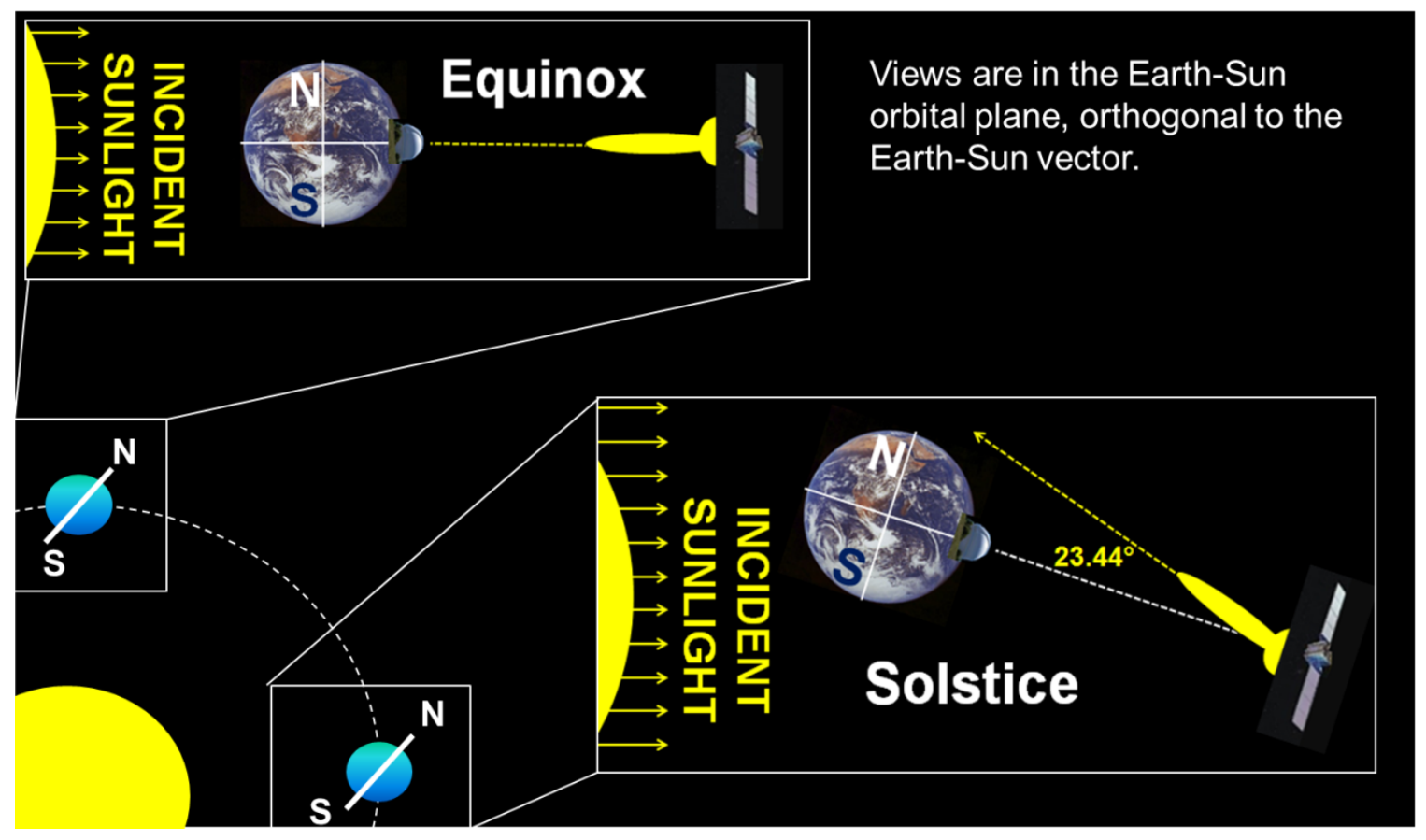

Figure 2: Schematic of the geometrical effect that results in a seasonal glint observed in the measured reflectance of a 3-axis stabilized GEO satellite, when measured at Equinox, Solstice, and other times of the year. The reflectance function of the satellite is represented by the yellow-colored shapes shown near the satellites. Near Equinox, peak reflectance from solar panels is expected to be observable.

The GOLDS campaign was designed to observe a set of eight active GEOs during a four week period coinciding with the winter 2013 glint season (February and March). Geographic diversity was achieved by employing contributing sensors at Maui, Kirtland AFB, Dayton, OH, Northern Virginia, and the US Air Force Academy (USAFA), both on campus in Colorado Springs and a mobile sensor located in northern Montana. Each sensor observed all objects during all nighttime hours on every clear night during the program. The USAFA sensors were tasked to examine details of the true glints to the greatest possible extent. As of the writing of this document, more than 50,000 magnitude observations were gathered and 35,000 of these are analyzed. Figure 1 provides a plot of the 3Dimensional surface of East/West phase angle, North/South phase angle, and visual magnitude in the Johnson B-filter for one of the eight satellites, DIRECTV-9S. The longitudinal diversity of the simultaneous GOLDS collects permits direct measure of the brightness as a function of North/South phase angle. Here the North/South phase angle is defined as the declination of the satellite less the declination of the sun. 


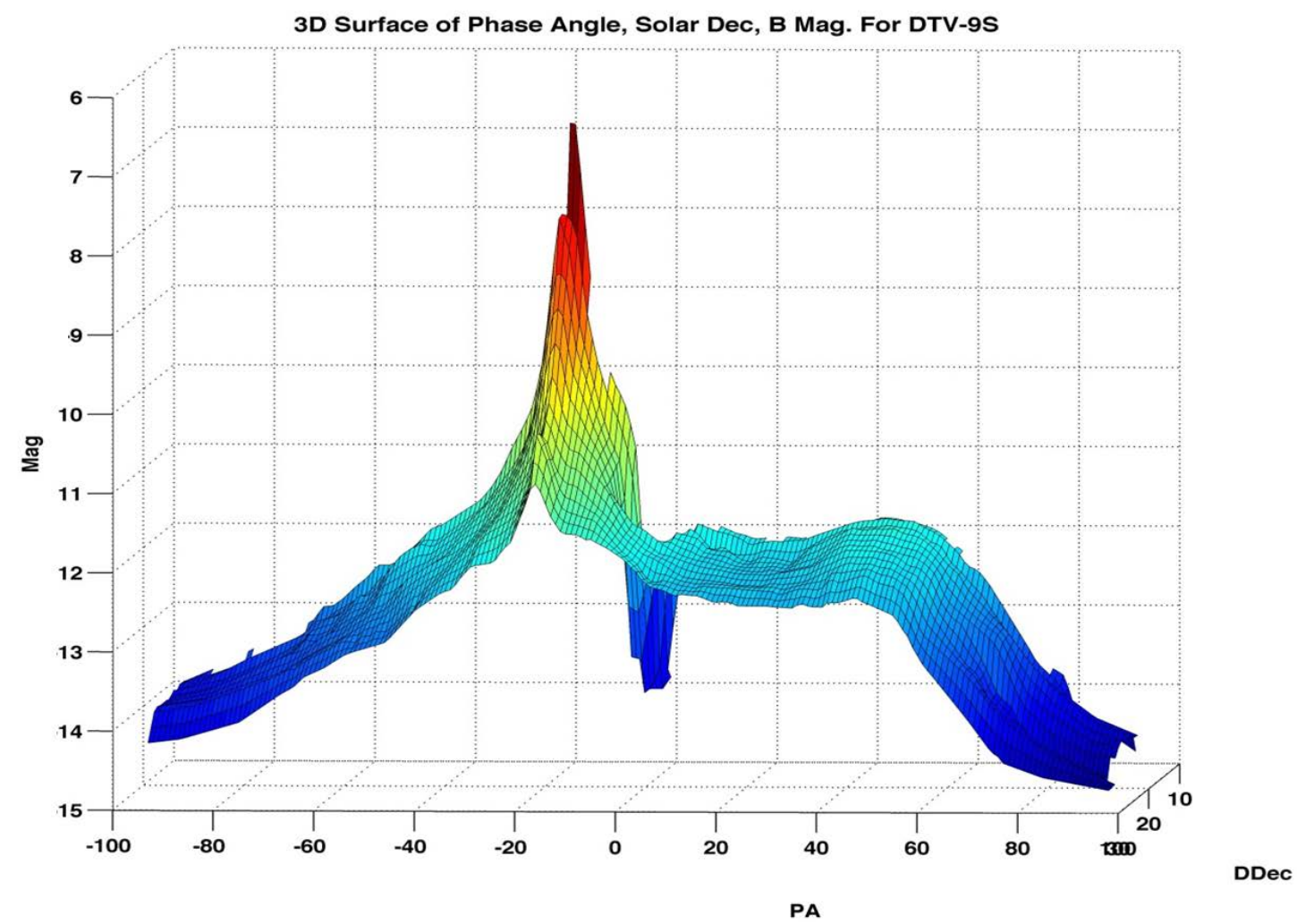

Figure 2: 3D plot of the brightness of DTV9S, as a function of East/West phase angle (PA), North/South phase angle (DDec). Phase angles go from left to right during a nighttime observing period and from front to back from January to March. Brighter values are lower magnitude than fainter values. Note the bright glint and the presence of the Earth’s shadow starting in Early March DDec 10.

Details of the distribution and number of peaks in plots such as that shown in Figure 2 are primary indicators of many types of SSA characterizations. The peaks are generally caused by near glint or true glints geometries with sunlight reflecting off solar panels. These uniquely identifiable characteristics such as the BRDF and pointing of the solar panel indicate power generation strategies of the satellite. As baseline measurements they hint at changes in satellite operation.

\section{GOLDS2}

The original GOLDS campaign observed the following GEOs: AMC-15, AMC-18, DTV4S, DTV-8, DTV-9S, DTV-10, DTV-12, and SES-1. We are very pleased with the amount and quality of the data. Early assessments indicate that the full analyses of these data sets will be of 
great SSA value. In addition to those SSA activities mentioned in Section I, we note that the extension of our understanding of the seasonal illumination effects will greatly enhance future Ibex-related programs that provide automated SSA analysis of GEOs. Furthermore, we expect to see minor differences in the data that are caused by different pose angles for each satellite as seen from sensors at different latitudes, and exploitation of these effects will help correlate small data features with small structures on the satellites.

Although GOLDS was successful, more collection campaigns are desired to continue improving and updating SSA knowledge. AFRL plans to conduct a GOLDS II campaign. Currently planned participants in this campaign are AFRL (RV, RD-Maui, and RY), USAFA and any other interested parties. Lessons learned during the GOLDS campaign suggest that for northerly sites it is preferable to partner with observatories that are equipped for cold weather. Thus, we plan to engage with academic observatories and have already identified a few potential partners. AFRL and USAFA plan to lead the campaign and direct the collects.

In addition to benefiting from lessons learned during the first GOLDS campaign, it is planned that we will pick a set of different GEOs to concentrate on; to include known and/or suspected rocket bodies, debris, or decommissioned/unstable objects. As characterization data of these class of objects is just a critical if not more so for Detect, Track, and ID. 


\section{Appendix H - Human Factors Survey}

\section{GOLDS Human Factors Site Survey}

Survey POC: John Ianni

$\underline{\mathrm{RH}}$ telescope lead: Dr. Vince Schmidt

\section{INTRODUCTION:}

Thank you for taking part in the GOLDS-II Human Factors Survey. This survey, developed the AFRL's Human Effectiveness Directorate (711 HPW/RH), is intended to help us evaluate human factors issues and best practices involved in a typical 30-day telescope data collection effort.

The intent is not to find fault with the procedures or approach used at any site. The responses will be and summarized and published in an AFRL technical report, and distributed to all contributors, with the hope that the report will contain some information of value to you in the execution of similar studies in the future.

Please include documentation for your procedures and process as requested in the survey. Your included procedures and process documentation will not be included in the final report without your permission. Findings will be described generally, without identifying specific sites, unless such information is valuable to the findings in the report and permissions are granted.

Please return the completed survey to john.ianni@us.af.mil by 30 November. You can also send comments/questions to this address any time.

\section{DEFINITIONS:}

Telescope Operator - A person capable of completely executing all telescope operations for a given study or collection effort. Requires a working knowledge of all equipment including telescope, camera, software, etc. For GOLDS-II, operators perform all system initialization and configuration actions preceding data collection, including telescope and imager setup and calibration. An operator's job is complete once the collection begins and is in a steady-state configuration.

Telescope Attendant - A person who monitors and maintains the system during data collection. Typical attendant tasks include ensuring valid camera exposure settings, monitoring weather, and perform other regular recording tasks. At some sites, attendants may be required to shut down the collector and facilities at the end of the scheduled collection period, or during inclement weather. Minimal training may be required to act as an attendant, but no previous astronomy-related experience is needed. 


\section{WORK ENVIRONMENT:}

Description of each telescope: optical and camera specifications, dome type, automation software, etc.

Environment for operators and attendants: co-located with telescope vs. separate control room, number and type of PCs and other equipment used during collection, etc.

\section{MANPOWER:}

How do you use operators and attendants? (How do they work together, did operators man the telescope all night, etc.?)

For sites with multiple telescopes, was there separate manning for each telescope or did one individual work all telescopes? Please outline any issues expected or encountered when manning multiple telescopes.

$+2$

How many personnel were available to be scheduled for the GOLDS-II collection effort? Without naming anyone, summarize background of these individuals (career, education, general background, etc.). How often did each individual participate?

\section{TRAINING:}

Describe the training operators receive (external classroom, self-taught, on-the-job, etc.)? Please provide any training documentation.

Describe the training attendants receive. Please provide any training documentation. 


\section{PROCEDURES:}

Please outline the nightly procedures followed by the operators (\& provide documentation if available).

Please outline the nightly procedures followed by the attendants ( $\&$ provide documentation if available).

For sites using multiple personnel each evening, how many personnel were used, and how much overlap in time occurred? Please describe the "handoff" procedures.

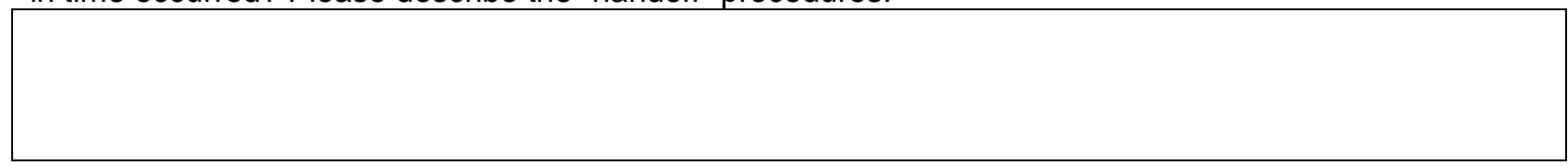

How do the procedures for the GOLDS-II collection effort differ from "typical" telescope usage?

\section{ISSUES:}

Describe any typical (expected or anticipated) problems attendants experienced during their shift? How were these problems mitigated?

Did attendants experience non-typical problems during their shift? How were these problems mitigated?

What actions did attendants take to prepare for their shift? 
Did attendants experience significant sleepiness or fatigue? If not, why not? If so, what did they do to reduce the impact of fatigue?

What times did attendants generally start and end their shifts? Describe typical commute times and distances.

\section{SHIFT:}

How long was a typical Operator shift?

How long was a typical Attendant shift, if applicable?

Estimate the percentage of time spent on these activities:

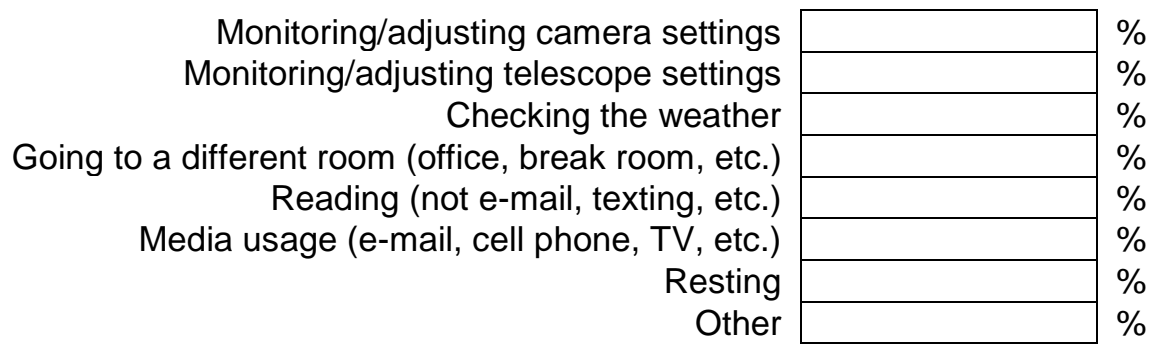

Comments about these percentages (if any):

\section{LESSONS LEARNED:}

Please describe lessons learned in preparation for, or during, this collection effort.

Distribution A: Approved for public release: distribution is unlimited. 88AB/PA Cleared 11/06/2014: 88ABW-2014-5151. 


\section{SUGGESTED CHANGES:}

Please indicate changes you would suggest or make (in any capacity) if this study were to be reexecuted.

\section{CHANGES IMPLEMENTED:}

Please describe any significant changes (in any capacity) that were suggested and made during the course of the study.

\section{OTHER:}

Please provide additional information that you think might be relevant to human factors issues, or to logistics issues, related to this study, particularly as such information applies to your site or configuration. 